\title{
Row-switched states in two-dimensional underdamped Josephson-junction arrays
}

\author{
Mauricio Barahona \\ Ginzton Laboratory, Stanford University, Stanford, California 94305
}

Shinya Watanabe*

Center for Chaos \& Turbulence Studies, Niels Bohr Institute, Blegdamsvej 17, Copenhagen, DK-2100, Denmark

(Received 21 October 1997; revised manuscript received 20 January 1998)

\begin{abstract}
When magnetic flux moves across layered or granular superconductor structures, the passage of vortices can take place along channels which develop finite voltage, while the rest of the material remains in the zerovoltage state. We study analytically an example of such mixed dynamics: the row-switched (RS) states in underdamped two-dimensional Josephson arrays, driven by a uniform dc current under external magnetic field but neglecting self-fields. The governing equations are cast into a compact differential-algebraic system which describes the dynamics of an assembly of Josephson oscillators coupled through the mesh current. We carry out a formal perturbation expansion, and obtain the dc and ac spatial distributions of the junction phases and induced circulating currents. We also estimate the interval of the driving current in which a given RS state is stable. All these analytical predictions compare well with our numerics. We then combine these results to deduce the parameter region (in the damping coefficient vs magnetic-field plane) where RS states cannot exist. [S0163-1829(98)11517-5]
\end{abstract}

\section{INTRODUCTION}

Two-dimensional (2D) arrays of Josephson junctions serve as "controlled laboratories" to investigate fundamental questions such as phase transitions, ${ }^{1}$ vortex propagation and interaction, ${ }^{2-5}$ phase and frequency locking of coupled oscillators, ${ }^{6-9}$ and spatiotemporal pattern formation and chaos, ${ }^{10,11}$ among others. ${ }^{12}$ A standard circuit geometry is a rectangular array driven by a dc current uniformly injected from the bottom and extracted from the top in the presence of an applied field (Fig. 1). Their technological promise as high-frequency oscillators ${ }^{13-15}$ depends critically on achieving tunable, highly nonlinear, coherent oscillations of the collection of junctions. However, such coherent oscillations are not easy to obtain, ${ }^{6,15,16}$ as the arrays frequently break up into incoherent substructures, and deliver output voltages with small ac amplitudes.

A striking example of such dynamical states with spatial structure is provided by the row-switched (RS) states found in underdamped 2D arrays of square cells. ${ }^{17}$ As the bias current $I_{\mathrm{dc}}$ is ramped up, the dc current-voltage characteristic $(I-V)$ of the array displays a succession of jumps between Ohmic branches of increasing resistance until, eventually, the normal resistive branch is reached. The observation that the branches are equally spaced in voltage suggested a rowswitching scenario, in which each jump corresponds to all the junctions in a row suddenly switching from the superconducting to the normal state, thus increasing the voltage across the array by a fixed amount. In the RS states, the array then consists of superconducting and normal rows, coexisting to form striped patterns as in the four examples shown in Fig. 2. In other words, the magnetic flux moves across the array along certain rows (channels) where a finite voltage develops, while the rest of the system remains in the zerovoltage state. This row-switching picture was later explicitly confirmed by measuring voltages across individual rows, ${ }^{18,19}$ and by direct imaging of the array. ${ }^{20}$

The row-switching phenomenon is robust to the change of the underlying lattice structure-in arrays of triangular cells it has been observed both experimentally ${ }^{21}$ and in simulations. $^{22}$ Thus, row switching could also be relevant to other systems. For instance, similar channeling of magnetic flux has been seen in continuous superconductors. ${ }^{23}$ Moreover, the hundreds of resistive steps which appear in the $I-V$ characteristics of high- $T_{c}$ superconductors ${ }^{24}$ have been taken as an indication of the layered weak-link structure in those materials.

The experiments on 2D arrays of square cells have re-

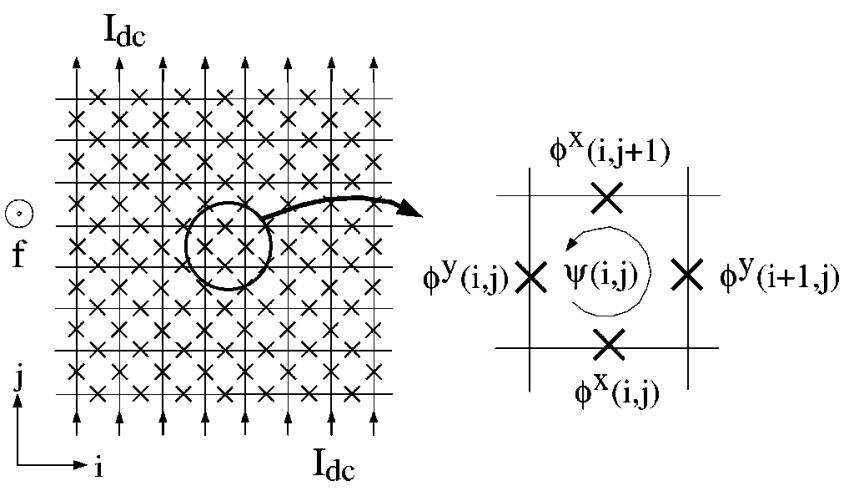

FIG. 1. 2D Josephson-junction array consisting of $N^{x}=7$ rows and $N^{y}=7$ columns of square cells. The cell at $(i, j)$ is shown enlarged. Each junction is described by a gauge-invariant phase difference: $\phi^{x}$ for the junctions on the horizontal edges, and $\phi^{y}$ for the vertical junctions. A uniform dc bias current $I_{\mathrm{dc}}$ is injected into every node on the bottom edge and extracted from the top. The left and right sides are open boundaries. The mesh current $\psi$ denotes the deviation of the current distribution from a uniform current flow in the vertical direction. A uniform magnetic field $f$, in units of the flux quantum $\Phi_{0}$, is applied normally to the plane of the array. 


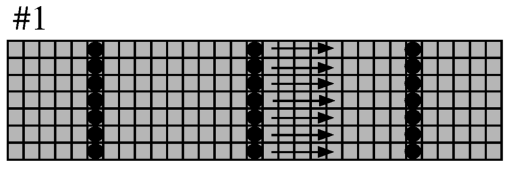

\#2

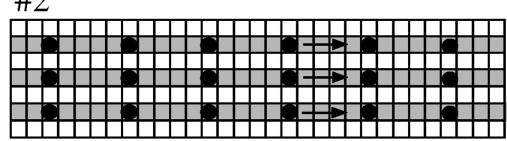

\#3

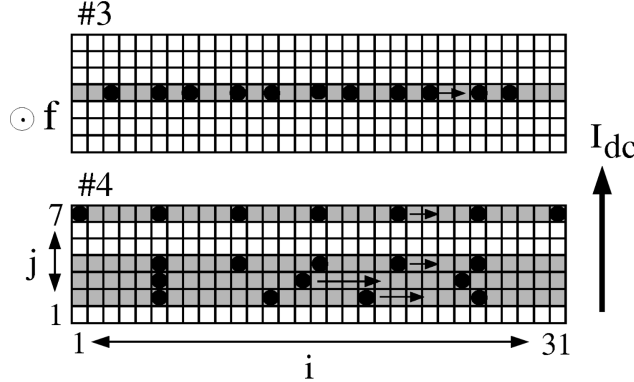

FIG. 2. Four snapshots of RS states in arrays of $N^{x}=31$ columns by $N^{y}=7$ rows. Two types of rows are observed: quiescent $(Q)$ rows (in white) across which there are zero dc voltage drops, and switched $(S)$ rows (shaded areas) across which there are finite dc voltage drops. Black dots denote topological vortices, defined in Sec. II. They are (roughly) equally spaced in the $S$ rows of the symmetric patterns $1-3$, but the spacing can change from row to row in asymmetric patterns such as pattern 4. Correspondingly, their propagation speed (represented by the length of the arrows) may change from an $S$ row to another within a pattern. In patterns 1 and 2, the vortices move in phase, even when the $S$ rows are separated by $Q$ rows. These patterns are numerically generated using $\Gamma=0.2, f=0.1$ and $I_{\mathrm{dc}}=0.6$ for patterns 1,2 , and 4 , and $I_{\mathrm{dc}}$ $=0.5$ in the case of pattern 3 . Thus, patterns 1,2 , and 4 correspond to coexisting dynamical attractors of the system.

vealed other properties of RS states. First, they only appear when the junctions are sufficiently underdamped. ${ }^{17,19,20,25}$ Otherwise, the $I-V$ characteristics present an extended region of flux-flow leading to the Ohmic branch of the entire array. Second, RS states are only observed for sufficiently small applied magnetic fields. If the field is too large, there are no individual RS steps; rather, one giant step emerges ${ }^{19,18}$ in the $I-V$. The origin of this giant step has been attributed to the interaction of self-fields with a coherent array oscillation in the form of a dynamical checkerboard pattern. ${ }^{26}$ Therefore, it is important to establish the parameter regime for the appearance of RS states and to determine their current and phase distributions in order to understand such transitions between coherent and localized states.

Much of the previous theoretical work on 2D arrays has consisted of numerical simulations ${ }^{27-30,4,22,2,31,32,18,25}$ which reproduce the measurements reasonably well. RS states have been discussed briefly, ${ }^{29,2}$ or more in depth ${ }^{22,32,18}$ in order to characterize the dynamics of the two types of rows found experimentally: (1) switched rows (" $S$ " rows), across which there is a finite dc voltage, and (2) quiescent rows (" $Q$ ", rows), across which there is no dc voltage drop. The simu- lations show that the junctions $\phi^{y}$ in the vertical branches of the $S$ rows are in the normal resistive state (rapidly rotating) whereas those in the $Q$ rows are nearly superconducting (stationary). Nevertheless, as we show, the junctions in the $Q$ rows are still oscillating, which causes finite ac voltage drops and associated losses. This is why we hesitate to call the $Q$ rows "superconducting."

Moreover, the numerical investigation ${ }^{22,18}$ of the rowswitching sequence shows that, even in the absence of temperature and disorder, the observed patterns and the order of their appearance depend on several factors: the initial condition, how the current is varied, the magnetic field (both externally applied and self-induced), etc. This is a clear indication that multiple attractors coexist for identical parameter values (as patterns 1, 2, and 4 in Fig. 2 show). When inhomogeneity in the junctions is included, it becomes even harder to predict which row will switch next, except to conjecture that it will occur at the "weakest part" of the array. ${ }^{22}$ Phillips et al. ${ }^{32}$ have studied the RS patterns in detail when inductances are included. When self-fields are small, the $S$ rows appear to be globally phase locked even if they are far apart, separated by $Q$ rows in between. This means that topological vortices in the $S$ rows appear to propagate together, just as seen in pattern 2 in Fig. 2. However, for generic asymmetric patterns, such as pattern 4 , vortices do not move together. Stronger self-fields are also found ${ }^{32}$ to break this phase coherence.

Compared to the numerous experimental and numerical studies, analytical results are much scarcer for $2 \mathrm{D}$ arrays. As far as we are aware, previous authors have focused on the simplest solution, namely, when the whole array is on the normal branch of the $I-V$ curve (pattern 1 in Fig. 2). This can be interpreted as the special RS state when all the rows have become normal; that is, the "completely row-switched" solution. These studies have concentrated on explaining the global phase-locking mechanism needed for oscillator applications. The complete RS state is found to be only neutrally stable under zero magnetic field ${ }^{6,33}$ (which implies that rows are decoupled), whereas a nonzero field induces interrow locking. These inter- and intrarow coupling mechanisms have been studied by several methods: isolating two cells in the array, ${ }^{34,7}$ perturbation methods, ${ }^{7}$ and harmonic balance. ${ }^{10,8}$ However, those results are not directly applicable to generic RS states, which exist only in a certain parameter regime, since the completely RS state extends to any large bias current for any damping.

In this paper, we study analytically the generic RS states and test our predictions against numerical integrations of the system. First, we cast the governing equations and the boundary conditions into a mesh formalism to ease the analytical procedure (Sec. II). In this notation, the system can be viewed as an array of coupled oscillators in which the junction phases $\phi$ (the pendulumlike oscillators) are coupled via the mesh currents $\psi$ (the current distribution in the array). The coupling arises from the flux quantization condition. We neglect self-field effects in the equations, thus reducing the parameters of the system to three: the bias current $I_{\mathrm{dc}}$, the junction damping coefficient $\Gamma$, and the magnetic field $f$. In this way, many properties of the RS states can be explained without undue complications. We also discuss the notion of vorticity in these discrete arrays. 
We use primarily four examples (depicted in Fig. 2) in order to illustrate and test our results. It is convenient to label each RS pattern by the set $S$ of its switched rows. Therefore, patterns 1 to 4 are labeled as $S=\{1,2,3,4,5,6,7\}, S=\{2,4,6\}$, $S=\{4\}$, and $S=\{2,3,4,7\}$, respectively. We also define an $S$ region to be a set of contiguous $S$ rows. For example, pattern 4 in Fig. 2 has two $S$ regions, one with three rows $2-4$ and another with a single row 7 . Similarly, a $Q$ region is a set of contiguous $Q$ rows.

A formal perturbation expansion in the high-frequency limit ${ }^{35}$ is used to analyze the governing equations (Sec. III). We assume that the RS states are time-periodic solutions in which some junctions whirl (i.e., the $\phi^{y}$ 's in the $S$ rows are running oscillators), and all the other junctions librate (i.e., the $\phi^{y}$ 's in the $Q$ rows and all $\phi^{x}$ are nearly stationary). Although the expansion is made systematic so that higherorder corrections could be obtained, we show that most of the features of the RS states can be accounted for by the leading order. (The only unresolved main feature is the phase locking between $S$ rows.) To the zeroth order, we obtain two systems of algebraic equations: one for the dc, and another for the ac components of the phases and currents. The dc system is nonlinear (thus difficult to solve); however, we obtain bulk approximations which work well far from the edges. On the other hand, the ac components obey the linear discrete Poisson equation with forcing from the dc solution; therefore, they can be readily obtained once the dc solution is known.

The bulk approximation determines analytically the dc and ac distributions of currents and phases for any given RS pattern. The first important result is that the dc current flows uniformly in the $S$ rows, but circulating currents are induced in the $Q$ regions. These strongly affect the spatial wave numbers of the $S$ rows (also calculated analytically), thus explaining why the spacing and speed of propagation of the fluxoids in the $S$ rows varies from pattern to pattern, and even from row to row within a pattern (Fig. 2). In Sec. IV we test these findings numerically with good agreement.

Another main conclusion from the leading-order analysis is that the presence of $S$ regions breaks the array into a collection of $Q$ regions that are decoupled from each other, as far as the dc equations are concerned. The $Q$ regions are, however, still weakly coupled through the ac component. Thus, for example, the existence of the switched row 4 in pattern 3 produces two $31 \times 3$ quasidisjoint $Q$ regions which only interact weakly. This picture proves useful because it reduces the problem of approximating the dynamical RS states of the array to obtaining the static states of its (smaller) constitutive $Q$ regions.

Indeed, this physical picture has further implications for the stability of the RS patterns (Sec. V). As explained above, each RS state is only observed in an interval of the bias current, which depends on the magnetic field and damping. We show that the upper current limit of this interval is well predicted by the depinning current of the largest $Q$ region. This means that the RS state ceases to exist when the flux penetrates any of the $Q$ regions which, in the absence of irregularities, is usually the largest one in the array. For example, pattern 3 cannot hold beyond the current at which a static state of a $31 \times 3$ array depins. Along the same lines, of all the RS states, the largest upper current will correspond to patterns whose largest $Q$ region is a single row, such as pattern 2.

However, this argument does not explain the simple observation: the RS states are found only in underdamped arrays, because the depinning currents are independent of the junction damping. This indicates that the lower current limit also plays an important role. A crude approximation for this lower limit is the retrapping current of a single junction which does depend on the damping, and it reproduces the numerics reasonably well. Combining the preceding criteria, we then calculate the region in the parameter plane of the magnetic field $f$ vs the damping parameter $\Gamma$ where $\mathrm{RS}$ states cannot exist. In accordance with experiments and simulations, we find that RS states occur most easily when both $\Gamma$ and $f$ are small. Throughout Sec. V we present additional numerical evidence to support these criteria.

\section{FORMULATION}

There are two equivalent ways of formulating the governing equations of the system: the node and mesh formulations. The node formulation is easier for simple geometries but it becomes cumbersome and impractical for two-dimensional arrays when inductances are included. Thus, we follow the previous literature, ${ }^{27,29,4,31,32,18}$ and derive a compact description of the arrays in the mesh formulation. In particular, we follow closely Phillips et al. ${ }^{31,32}$ and Trías, ${ }^{18}$ with a few changes. Although this formalism was originally developed to ease numerical simulations, it is well suited for analytical work.

\section{A. Governing equations}

Our description of the array shown in Fig. 1 assumes several simplifications. First, we neglect thermal fluctuations (i.e., zero temperature), and we consider all junctions identical (i.e., no disorder). Second, we describe our basic circuit unit, a single Josephson junction, by the resistively and capacitively shunted junction model. In this standard model, a junction driven by a current $I^{b}$ is represented by an equivalent circuit of three channels in parallel with a capacitance $C$, a resistance $R$, and a tunnel junction with the critical current $I_{c}$. As a result, its state variable $\phi$ (the gauge-invariant phase difference across the junction) is governed by

$$
\mathcal{N}[\phi] \equiv \ddot{\phi}+\Gamma \dot{\phi}+\sin \phi=I^{b},
$$

where the nonlinear operator $\mathcal{N}$ returns the total current through the device. In Eq. (1) the current is normalized by $I_{c}$, whereas time is expressed in units of the inverse of the plasma frequency $\omega_{p}^{-1}=\left(\Phi_{0} C / 2 \pi I_{c}\right)^{1 / 2}$. In addition, $\Gamma$ $=\beta_{c}^{-1 / 2}=\left(\Phi_{0} / 2 \pi I_{c} R^{2} C\right)^{1 / 2}$ is the damping, with $\beta_{c}$ the McCumber parameter. Also, $\Phi_{0}$ is the quantum of magnetic flux. The instantaneous voltage across the junction is given by the Josephson voltage-phase relation:

$$
V(t)=\Gamma \dot{\phi},
$$

where the voltage is normalized by $I_{c} R$. Thus, a single junction is analogous to a damped-driven mechanical pendulum, and its voltage corresponds to the rotation frequency of the pendulum. $^{35-37}$ 
When several junctions are interconnected to form a network, like the one depicted in Fig. 1, the current distribution must fulfill Kirchhoff's current law. This results in coupling among the junctions. It is convenient to decompose each branch current into an external and a deviation current:

$$
I^{b}=I_{\mathrm{ext}}+I_{\mathrm{dev}} .
$$

The external current $I_{\text {ext }}$ is chosen such that it satisfies current conservation at all nodes, including external sources and sinks. In general, it can be spatially nonuniform or time dependent. However, as the steady bias current $I_{\mathrm{dc}}$ is injected (extracted) at the nodes along the bottom (top) edges, our choice $^{38}$ for $I_{\text {ext }}$ is the stationary uniform vertical flow, in which $I_{\mathrm{ext}}=I_{\mathrm{dc}}$ on every vertical branch of the circuit (for all $t$ ), and $I_{\mathrm{ext}}=0$ on every horizontal bond.

The deviation from the external flow $I_{\mathrm{dev}}$ must be divergence free since current sources and sinks have already been incorporated into $I_{\text {ext }}$. Therefore, there exists a stream function (or mesh current) $\psi$ at each cell whose discrete curl determines $I_{\mathrm{dev}}$ in the $x$ and $y$ directions:

$$
\begin{gathered}
I_{\mathrm{dev}}^{x}(i, j)=\psi(i, j)-\psi(i, j-1), \\
I_{\mathrm{dev}}^{y}(i, j)=-[\psi(i, j)-\psi(i-1, j)] .
\end{gathered}
$$

(In the rest of this paper we will not write time dependences explicitly when they are obvious, such as here.)

In order to ensure that these relations hold also at the edges of the array, we define artificial boundary cells which have either the horizontal index $i=0$ or $N^{x}+1$, or the vertical index $j=0$ or $N^{y}+1$. This yields the boundary conditions of the problem:

$$
\psi(i, j)=0 \quad \text { if } i=0, N^{x}+1 \quad \text { or if } j=0, N^{y}+1 .
$$

This condition is equivalent to "grounding" the value of $\psi$ outside the array.

Combining Eqs. (1)-(5), we obtain the first two sets of governing equations

$$
\begin{gathered}
\left.\mathcal{N} \phi^{x}(i, j)\right]=\psi(i, j)-\psi(i, j-1), \\
\mathcal{N}\left[\phi^{y}(i, j)\right]=I_{\mathrm{dc}}-[\psi(i, j)-\psi(i-1, j)],
\end{gathered}
$$

where $\mathcal{N}$ was defined in Eq. (1).

The other source of intrinsic coupling between the junctions is due to a macroscopic quantum constraint: the flux quantization condition around each cell. Given that the corners of each cell are superconducting islands described by well-defined phases, the phase change around cell $(i, j)$ yields the third and final set of equations of the system

$$
\begin{aligned}
& {\left[\phi^{y}(i+1, j)-\phi^{y}(i, j)\right]-\left[\phi^{x}(i, j+1)-\phi^{x}(i, j)\right]} \\
& \quad+2 \pi \frac{\Phi(i, j)}{\Phi_{0}}=2 \pi n(i, j)
\end{aligned}
$$

for $i=1, \ldots, N^{x}$ and $j=1, \ldots, N^{y}$, where $\Phi(i, j)$ is the total magnetic field penetrating the cell. The winding numbers $n(i, j)$ are a set of integers that arise because the island phases are only defined up to multiples of $2 \pi$. The $n(i, j)$ are fixed by the initial condition and remain constant as long as the array is kept superconducting. However, without loss of generality, all $n(i, j)$ can be set to zero. Suppose they are not zero; then we can redefine the junction phases as

$$
\begin{gathered}
\phi^{x}(i, j) \rightarrow \phi^{x}(i, j), \\
\phi^{y}(i, j)-2 \pi \sum_{k=1}^{i-1} n(k, j) \rightarrow \phi^{y}(i, j),
\end{gathered}
$$

such that Eq. (9) is unchanged except, now, all $n(i, j) \equiv 0$. Crucially, both currents and voltages are invariant under this redefinition of the phases since adding integer multiples of $2 \pi$ to $\phi^{y}$ changes neither $\sin \phi^{y}$ nor $\dot{\phi}^{y}$. This means that the dynamics and measurements remain identical for any combination of integers $n(i, j)$, and we do not need to be concerned with their initial values. Similarly, if the magnetic field were controllable independently on each cell, adding an integer number of flux quanta $\Phi_{0}$ into any cell would not change the measured $I-V$ characteristics, at least within this model. This is simply the array analog of the two-junction superconducting quantum interference device, whose dependence on the penetrating field is also $\Phi_{0}$ periodic. Because of this periodicity in the magnetic field, the topological vortex must be defined differently in 2D arrays and in continuous superconductors, as we will discuss at the end of this section.

The total magnetic field in Eq. (9) can be decomposed into two parts:

$$
\Phi(i, j)=\Phi_{\text {ext }}+\Phi_{\text {ind }}(i, j) .
$$

The first term is produced by the external field applied perpendicularly to the plane of the array, which we assume to be constant and uniform. It is usually parametrized as a dimensionless frustration $f$ normalized to the flux quantum:

$$
f=\Phi_{\text {ext }} / \Phi_{0},
$$

such that, in terms of $f$, the period of the external field is unity. The second term, the induced field, can be written generally as the sum of all the contributions from the branch currents

$$
\Phi_{\text {ind }}(i, j)=\sum_{n} \sum_{k} L_{n, k}^{b} I_{k}^{b},
$$

where $k$ runs through all the branches of the circuit, while $n$ corresponds to the four edges of cell $(i, j)$. The branch inductances $L_{n, k}^{b}$ are purely geometric constants determined from the circuit. ${ }^{4,31}$

\section{B. Matrix-vector notation}

The above equations can be cast into a compact matrixvector notation. ${ }^{31}$ For a $N^{x} \times N^{y}$ array, all branch variables (e.g., currents $I^{b}$, voltages $V$, and phases $\phi$ ) can be written as vectors of dimension equal to the number of branches, i.e., $\left(N^{x}+1\right) N^{y}+N^{x}\left(N^{y}+1\right)$. Thus, for instance, the vector $\phi$ consists of all the phases $\phi^{x}$ and $\phi^{y}$. On the other hand, variables defined at cells (e.g., the mesh current $\psi$ and the induced flux $\Phi_{\text {ind }}$ ) form vectors of dimension $N^{x} N^{y}$. These two groups of vectors are connected via a branch-to-cell connectivity matrix ${ }^{39} M$ which takes a directed sum as we loop around a cell: 
$M \phi(i, j)=\left[\phi^{y}(i+1, j)-\phi^{y}(i, j)\right]-\left[\phi^{x}(i, j+1)-\phi^{x}(i, j)\right]$.

More mathematically, this operator takes the discrete curl of $\phi$ around every cell $(i, j)$. Conversely, the discrete curl of the cell variables is obtained by applying the transposed cellto-branch matrix $M^{T}$

Using this notation, the total flux (11) can be written as

$$
\Phi=\Phi_{0} f+M L^{b} I^{b},
$$

where $L^{b}$ is the branch inductance matrix, and $f$ is a constant vector.

Moreover, Eqs. (4),(5) can now be written simply as

$$
I_{\mathrm{dev}}=M^{T} \psi
$$

and Eqs. (7),(8) become

$$
\mathcal{M}[\phi]=I_{\text {ext }}+M^{T} \psi,
$$

where $\mathcal{N}$ is operated component wise and the vector $I_{\text {ext }}$ has components that are zero on the horizontal edges and $I_{\mathrm{dc}}$ on the vertical edges, as defined by our choice of stationary uniform vertical flow.

Finally, we can use Eqs. (14) and (15) to recast the flux quantization condition (9) as

$$
M \phi+2 \pi f+\frac{1}{\lambda_{\perp}}\left(L^{m} \psi+M L^{b} I_{\text {ext }}\right)=0,
$$

where components of $L^{b}$ are normalized to $\mu_{0} p, p$ is the lattice constant, $\lambda_{\perp}=\Phi_{0} / 2 \pi I_{c} \mu_{0} p$ is the dimensionless penetration depth, the mesh inductance matrix is defined as

$$
L^{m}=M L^{b} M^{T},
$$

and we have set $n(i, j) \equiv 0$.

To summarize, the governing equations (17) and (18) form a closed differential-algebraic system for $\phi$ and $\psi$, with parameters $f, \Gamma, I_{\mathrm{dc}}, \lambda_{\perp}$, and the coefficient matrix $L^{b}$. This form of the system is compact and intuitive. It can be seen as a coupled-oscillator system in which the "oscillators" $\phi$ are driven by the "coupling field" $\psi$ in Eq. (17). In return, the oscillators collectively feed back onto the field in Eq. (18). This picture suggests the following integration steps: ${ }^{32,18}$ first, given $\phi$ at some time $t$, solve Eq. (18) for $L^{m} \psi$; then, invert the matrix $L^{m}$, together with the boundary conditions (6), to determine the field $\psi$. This gives us the "drive" on the right-hand side of Eq. (17), which is used to update the oscillators $\phi$ in time.

We conclude the general formulation by pointing out that Eqs. (17), (18) possess two simple symmetries. ${ }^{29}$ If we find a solution $[\phi(i, j, t), \psi(i, j, t)]$ for $f$ and $I_{\mathrm{dc}}$, then $[-\phi(i, j, t),-\psi(i, j, t)]$ is a solution of the system for $-f$ and $-I_{\mathrm{dc}}$, the other parameters being the same. Similarly, $[-\phi(-i,-j, t), \psi(-i,-j, t)]$ is also a solution for $f$ and $-I_{\mathrm{dc}}$ (since $M$ is changed to $-M$ due to the reversal of the spatial coordinates). Therefore, we only have to study the quadrant $f \geqslant 0$ and $I_{\mathrm{dc}} \geqslant 0$. Together with the unit periodicity in $f$, the frustration can be further restricted to $0 \leqslant f<1 / 2$ without loss of generality. Thus, in the rest of this article, by "large $f$ " and "small $f$ " we mean frustration values close to $1 / 2$ and 0 , respectively.

\section{No-inductance approximation}

Computing the full equations (17),(18) quickly becomes a heavy task as the system size increases. In previous numerical studies, these computational limitations have been circumvented either by using acceleration schemes ${ }^{4,31}$ when the inductance effects are of interest per se, or by "truncating", the matrix $L^{m}$ (i.e., neglecting some of its components). Three truncations $s^{4,31,32,18}$ are often used: no-, self-, and nearest-neighbor inductances. Self-inductance neglects the intercell magnetic coupling by keeping only the diagonal components of $L^{m}$ (which then becomes trivially invertible). Nearest-neighbor inductance includes, in addition, magnetic coupling between neighboring cells. An important remark is that not only the mesh inductance $L^{m}$ but also the vector $M L^{b} I_{\text {ext }}$ must be provided in order to complete the system, and the choice of $I_{\text {ext }}$ may affect the results when $L^{b}$ is truncated. ${ }^{4,18}$ (In contrast, the choice of $I_{\text {ext }}$ is unrestricted if the full inductance matrix is used.) Truncating the system in a physically consistent manner is a subtle issue, and, for simplicity, we shall assume no inductance in this article.

In contrast to what one might guess from its name, the no-inductance approximation does not eliminate the intercell coupling. Counterintuitively, it leads to an even longer-range coupling than the self- and nearest-neighbor truncations. The no-inductance approximation sets $L^{b}=0$, thus $L^{m}=0$. The flux quantization condition (18) is then just

$$
M \phi+2 \pi f=0 \text {. }
$$

The same equation can also be obtained in the limit $\lambda_{\perp}=\infty$ for any $L^{b}$, which allows the no-inductance limit to be approached from the inductive system continuously. It is important to note that the condition (20) is now a constraint on $\phi$, which must be satisfied at all times. The discrepancies between $M \phi$ and $-2 \pi f$ cannot be filled by locally adjusting the induced field, as when the inductive terms were present. This leads to a global coupling of the junctions over the whole domain. To see the coupling mechanism provided by Eq. (20), operate the loop sum $M$ on Eq. (17). From the left-hand side of Eq. (17) we obtain

$$
M \mathcal{N}(\phi)(i, j)=M \ddot{\phi}+\Gamma M \dot{\phi}+M[\sin \phi]
$$

but the first two terms vanish, since Eq. (20) must hold at all times. From the right-hand side of Eq. (17) we obtain

$$
M\left(I_{\mathrm{ext}}+M^{T} \psi\right)=M I_{\mathrm{ext}}+M M^{T} \psi=M I_{\mathrm{ext}}-\Delta \psi,
$$

where we have introduced the discrete Laplacian

$$
\begin{aligned}
\Delta \psi(i, j) \equiv & {[\psi(i, j+1)+\psi(i, j-1)+\psi(i+1, j)+\psi(i-1, j)] } \\
& -4 \psi(i, j) .
\end{aligned}
$$

For the stationary uniform flow $I_{\text {ext }}$, the term $M I_{\text {ext }}=0$. Thus, we arrive at a discrete Poisson equation

$$
\Delta \psi=-M[\sin \phi],
$$

in which the distribution of the mesh current is dependent on all the junctions in the array.

Equations (17) and (22) constitute the governing equations for the no-inductance case, and can be integrated as before. Provided that the initial condition satisfies the con- 
straint (20) and its time derivative $M \dot{\phi}=0$, Eq. (20) is satisfied for all $t$. An immediate advantage of the no-inductance approximation is that the sweep of the parameter space is greatly simplified since the number of parameters has been reduced to three: $f, \Gamma$, and $I_{\mathrm{dc}}$.

\section{Vorticities in 2D arrays}

Before closing this section, we consider now the concept of vorticity in these arrays. As for incompressible planar fluid flows, we can define a vorticity by taking the curl (by applying $M$ ) of the "velocity" field which, in our case, corresponds $^{39}$ to the branch current $I^{b}$. This current vorticity

$$
\Omega=M I^{b}=M\left(I_{\mathrm{ext}}+M^{T} \psi\right)=M I_{\mathrm{ext}}-\Delta \psi
$$

measures how currents whirl, and can take any real values. (For our stationary uniform vertical flow $M I_{\text {ext }}$ vanishes.)

In contrast, the notion of a topological vortex (or charge) is commonly used in Josephson arrays in analogy to continuous superconductors. In type-II superconductors, the vortices would correspond to the integer winding numbers $n(i, j)$ in the flux quantization condition (9). But, as we showed above, the $n(i, j)$ are dynamically irrelevant in the arrays. Therefore, an alternative, less physical definition for the topological vorticity has been used: $2,4,32,18$

$$
\zeta=\frac{1}{2 \pi} M(\hat{\phi}-\phi)
$$

Here, $\hat{\phi}$ denotes restriction of the components of the phase vector $\phi$ within $[-\pi, \pi)$. The value of $\zeta$ at each cell takes only integer values (typically 0 or \pm 1 ) and jumps discontinuously as the system evolves in time. In effect, this definition detects when one of the four junctions in a cell rotates and crosses $\phi=\pi(\bmod 2 \pi)$, since $M \hat{\phi}$ changes discontinuously by $2 \pi$ at that instant. This is the $2 \mathrm{D}$ analog of marking the location of a 1D kink at the point where $\phi=\pi(\bmod 2 \pi)$ regardless of whether the kink really has a localized structure or not. This particlelike picture is frequently useful but, by neglecting spatial distributions, there is a potential loss of information about the true dynamical state of the system. On the other hand, the current vorticity $\Omega$ would reveal more accurately how localized vortices are. However, for the RS states treated here, our simulations show that the topological vorticity $\zeta$ moves together with a peak of the current vorticity $\Omega$ (Sec. IV B). Thus, we use both definitions interchangeably at our convenience.

\section{ANALYSIS}

In this section we present a perturbative analysis of the governing equations (17),(20). Although the analysis is made systematically so that it is possible to proceed to higher orders, we show that most of the fundamental features of the row-switched states can be explained by the leading order of the expansion.

Before writing down the RS solutions, it is useful to think of the array with uncoupled junctions. This limiting case corresponds to imposing $\psi=0$ in Eq. (17), thus reducing the array to a collection of uncoupled pendula, independently responding to a constant drive. The junctions on the horizon- tal branches (7), whose drive is zero, converge asymptotically to $\phi^{x *}=0$ due to the damping. On the other hand, the uncoupled vertical junctions $(8)$, driven by $I_{\mathrm{dc}}$, have a different dynamical behavior. For small damping $\Gamma$, they can converge asymptotically to one of two distinct stable states: ${ }^{36}$ the superconducting (static) state, which exists only when $I_{\mathrm{dc}}<1$, in which the drive is balanced by the sinusoidal nonlinearity, (i.e., $\phi^{y *}=\arcsin I_{\mathrm{dc}}$ ); or the Ohmic (whirling) solution, where the first time derivative balances the drive, and $\phi$ increases at a nearly uniform rate $\omega=I_{\mathrm{dc}} / \Gamma$ (i.e., the pendulum "whirls"). The two attractors may coexist for the same drive, and hysteresis may occur.

When the junctions are coupled, the simple dynamics of the independent junctions is altered, and complex spatiotemporal solutions, which do not have an analog in the uncoupled array, may emerge. Nevertheless, in the case of the RS solutions the two states of the driven single junction mentioned above (static and whirling) are still valuable "building blocks", for the analysis of the whole system. Specifically, the RS states are characterized by alternating regions in which the vertical junctions are either stationary ( $Q$ regions) or whirling ( $S$ regions). There are, however, some significant differences with the uncoupled case. For instance, the time-averaged current distribution in the coupled array deviates from the uniform flow. Hence, the phases of the stationary junctions can have other values than 0 or $\arcsin I_{\mathrm{dc}}$. In addition, the rotations of the vertical whirling junctions induce ac oscillations on the stationary junctions, and phase locking among the whirling junctions. Our analysis in this section is capable of explaining most of these effects.

We note that our analysis is restricted to solutions with no (static) vortices trapped in any of the $Q$ regions. The "novortex" state is expected to be most relevant to determine the parameter regime in which RS states appear. Similarly, the vertical junctions in the $S$ regions are assumed to be whirling at almost constant frequency. More nonlinear rotations are certainly possible as we briefly discuss in Sec. VI, but are neglected.

\section{A. Perturbative analysis}

In previous perturbative analyses of junctions and arrays, it has been customary to treat $I_{\mathrm{dc}}$ as a large parameter. ${ }^{7,40}$ However, partially RS states can exist only when $I_{\mathrm{dc}}$ is sufficiently small, as we will show below. Therefore, we use the rotation frequency of the pendulum $\omega=I_{\mathrm{dc}} / \Gamma$ as the large parameter in our perturbation. That is, we will consider the high-frequency limit ${ }^{35} \omega \gg 1$, which can be satisfied for any finite $I_{\mathrm{dc}}$ if the damping $\Gamma$ is small enough.

Hence, we assume that the variables in the RS states can be expanded in powers of $\omega^{-1}$. The phases of the horizontal junctions are then approximated by

$$
\phi^{x}(i, j, t)=\overline{\phi_{0}^{x}}(i, j)+\sum_{p=2}^{\infty} \omega^{-p} \phi_{p}^{x}(i, j, \tau),
$$

while the mesh current is given by

$$
\psi(i, j, t)=\overline{\psi_{0}}(i, j)+\widetilde{\psi_{0}}(i, j, \tau)+\sum_{p=1}^{\infty} \omega^{-p} \psi_{p}(i, j, \tau),
$$


where we have introduced the normalized time

$$
\tau=\omega t=\left(I_{\mathrm{dc}} / \Gamma\right) t
$$

The notation $(\bar{\cdot})$ expresses time-independent $(\mathrm{dc})$ quantities, while $(\widetilde{\cdot)}$ are for the time-dependent (ac) parts whose time average is zero. Note that the correction of $O\left(\omega^{-1}\right)$ in Eq. (25) turns out to be zero, so we neglect that term from the start.

The form for the vertical junctions must be different in the switched and the quiescent rows. In the switched rows, all the junctions are whirling and stationary: their phases grow, to the lowest order, constantly in time

$$
\phi^{y}(i, j, t)=\tau+\overline{\phi_{0}^{y}}(i, j)+\sum_{p=2}^{\infty} \omega^{-p} \phi_{p}^{y}(i, j, \tau), \quad j \in S .
$$

Meanwhile, in the quiescent rows the junctions are librating around fixed values, and thus, the leading order is independent of time:

$$
\phi^{y}(i, j, t)=\overline{\phi_{0}^{y}}(i, j)+\sum_{p=2}^{\infty} \omega^{-p} \phi_{p}^{y}(i, j, \tau), \quad j \in Q .
$$

We impose $\widetilde{\psi_{0}}$ and the higher-order terms to be periodic in time. In general, the period has to be modulated and, thus, expanded in $\omega^{-1}$ (strained coordinate). However, since in the following we will focus on the leading order system, we set the period to be exactly $2 \pi$ in $\tau$ for simplicity.

The perturbative calculation proceeds in the usual way by substituting Eqs. (25)-(28) into Eqs. (17),(20); Taylor expanding the sine in Eq. (1); and equating terms of the same order in $\omega$. In principle, this procedure can be carried out to higher orders if secular terms are eliminated by satisfying solvability conditions when they arise.

Balancing the leading-order terms, we obtain two sets of equations since the time-independent (dc) and timedependent (ac) terms must cancel separately. First, the dc terms yield the following equations for both types of rows:

$$
\begin{gathered}
\sin \overline{\phi_{0}^{x}}(i, j)=\overline{\psi_{0}}(i, j)-\overline{\psi_{0}}(i, j-1), \\
\overline{\phi_{0}^{x}}(i, j+1)-\overline{\phi_{0}^{x}}(i, j)=2 \pi f+\overline{\phi_{0}^{y}}(i+1, j)-\overline{\phi_{0}^{y}}(i, j),
\end{gathered}
$$

and one more equation which depends on the type of row (switched or quiescent):

$$
\begin{gathered}
0=\overline{\psi_{0}}(i, j)-\overline{\psi_{0}}(i-1, j), \quad j \in S, \\
I_{\mathrm{dc}}-\sin \overline{\phi_{0}^{y}}(i, j)=\overline{\psi_{0}}(i, j)-\overline{\psi_{0}}(i-1, j), \quad j \in Q .
\end{gathered}
$$

These equations constitute the full dc system.

Similarly, from the ac terms we obtain for both rows

$$
\begin{gathered}
\phi_{2}^{x^{\prime \prime}}(i, j, \tau)=\widetilde{\psi_{0}}(i, j, \tau)-\widetilde{\psi_{0}}(i, j-1, \tau), \\
\phi_{2}^{x}(i, j+1, \tau)-\phi_{2}^{x}(i, j, \tau)=\phi_{2}^{y}(i+1, j, \tau)-\phi_{2}^{y}(i, j, \tau),
\end{gathered}
$$

where " denotes differentiation twice with respect to $\tau$. Moreover, for each type of row we obtain a different equation:

$$
\begin{aligned}
\phi_{2}^{y^{\prime \prime}}(i, j, \tau)= & -\sin \left(\tau+\overline{\phi_{0}^{y}}(i, j)\right)-\widetilde{\psi_{0}}(i, j, \tau) \\
& +\widetilde{\psi_{0}}(i-1, j, \tau), \quad j \in S
\end{aligned}
$$

and

$$
\phi_{2}^{y^{\prime \prime}}(i, j, \tau)=-\widetilde{\psi_{0}}(i, j, \tau)+\widetilde{\psi_{0}}(i-1, j, \tau), j \in Q,
$$

which completes the full ac system.

These systems of equations are to be solved with boundary conditions

$$
\overline{\psi_{0}}=\widetilde{\psi_{0}}=0
$$

at the boundary cells.

A simple but important observation can be made at this point. Using Eqs. (31) and (37) at $i=0$ and $N^{x}+1$ (i.e., at the right and left edges), it follows that

$$
\overline{\psi_{0}}(i, j)=0 \quad \forall i, \quad \text { if } j \in S .
$$

Therefore, the leading-order dc mesh current vanishes in a switched row, ${ }^{43}$ just as it does in the top and bottom boundary cells at $j=0$ and $N^{y}+1$. In other words, the switched row is equivalent to having another boundary row, which splits the array into two. Thus, to the leading order, a partially row-switched array with many switched rows can be described as a collection of disjoint quiescent regions, coupled only weakly through the ac component. This useful picture is exploited later.

The solution of the leading-order systems is otherwise nontrivial since the dc equations (29)-(32) constitute a nonlinear algebraic system, and the dc solution is in turn needed to solve the ac system (33)-(36). Thus, in general, they have to be solved numerically - although we show below that useful approximations can be obtained under certain assumptions.

Once the leading-order solutions are found, the calculation could be carried out to higher orders. The next-order correction leads to a particularly simple set of equations:

$$
\begin{gathered}
\phi_{3}^{x \prime \prime}(i, j)+\Gamma \phi_{2}^{x \prime}(i, j)=\psi_{1}(i, j)-\psi_{1}(i, j-1), \\
\phi_{3}^{y \prime \prime}(i, j)+\Gamma \phi_{2}^{y \prime}(i, j)=-\psi_{1}(i, j)+\psi_{1}(i-1, j), \\
\phi_{3}^{x}(i, j+1)-\phi_{3}^{x}(i, j)=\phi_{3}^{y}(i+1, j)-\phi_{3}^{y}(i, j),
\end{gathered}
$$

for all $\tau$ and regardless of whether the row $j$ is switched or quiescent. Terms from the sinusoidal nonlinearity do not come into play at this order, but further expansions would certainly involve more complications.

It is important to note, however, that the salient features of the solutions observed in the numerics can be explained from the leading-order equations. Therefore, we restrict our analysis to the dc and ac equations in the following sections. On the other hand, we will also point out a remaining problem which is likely to be resolved only by considering the higher-order corrections. 


\section{B. Analysis of the de equations}

The dc equations (29)-(32) constitute a nonlinear algebraic system which must be solved numerically in general. However, to gain insight into the system, we will now obtain approximate solutions to the system when there is a large asymmetry between its two dimensions. We will then come back to the full system and discuss its solutions.

\section{Large aspect-ratio approximation}

Consider the case when all quiescent regions in the array are longer horizontally than vertically. This happens, of course, when the array itself satisfies $N^{x} \gg N^{y}$. More importantly, arrays whose dimensions do not fulfill this condition are also broken into smaller, laterally long, almost disjoint quiescent regions after several row-switching events. Thus, this "large aspect-ratio", approximation is important to characterize the RS states which appear in the course of the rowswitching process. Remember we also assume that none of the $Q$ regions contains static vortices, which could be trapped for large $N^{y}$ and $f$, and for small $I_{\mathrm{dc}}$. In Sec. V we will give an estimate of the values of $f$ and $N^{y}$ for which we expect this assumption to be valid.

In such a situation we expect a nearly "uniform" solution in the bulk of the array with some edge corrections near the right and left boundaries. Hence, far from the boundaries, we assume the vertical junctions in the quiescent rows to become independent of $i$,

$$
\overline{\phi_{0}^{y}}(i, j)=\overline{\phi_{0}^{y}}(j) \quad \text { for } j \in Q .
$$

On the other hand, we assume a whirling solution ${ }^{41}$ for the switched rows in which waves with well-defined wave numbers $k(j)$ propagate:

$$
\overline{\phi_{0}^{y}}(i, j) \approx-k(j) i+\delta(j) \quad \text { for } j \in S .
$$

Note that the wave number $k(j)$ and the phase constant $\delta(j)$ may differ from one switched row to another. The other dc variables $\overline{\phi_{0}^{x}}$, and $\overline{\psi_{0}}$ are also assumed to be $i$ independent.

Thus, the de equations reduce to

$$
\begin{gathered}
\sin \overline{\phi_{0}^{x}}(j)=\overline{\psi_{0}}(j)-\overline{\psi_{0}}(j-1), \\
\overline{\phi_{0}^{x}}(j+1)-\overline{\phi_{0}^{x}}(j)=2 \pi f-k(j) \quad \text { for } j \in S, \\
\overline{\phi_{0}^{x}}(j+1)-\overline{\phi_{0}^{x}}(j)=2 \pi f \quad \text { for } j \in Q, \\
\overline{\psi_{0}}(j)=0 \text { for } j \in S, \\
\overline{\phi_{0}^{y}}(j)=\arcsin I_{\mathrm{dc}} \quad \text { for } j \in Q .
\end{gathered}
$$

This simplified set of equations is still nonlinear but solvable. We begin by analyzing all the quiescent regions in the array (if any), delimited by switched regions or by the physical boundaries. Consider a quiescent region spanning from row $j_{1}$ to $j_{2}\left(\geqslant j_{1}\right)$. Such a region contains $n=j_{2}-j_{1}+2$ rows of horizontal junctions including the top and bottom borders, and $n-1$ quiescent rows of vertical junctions. We emphasize that these vertical phases are all given by Eq. (47), thus, $I_{\mathrm{dc}}<1$ is necessary for the existence of partially
RS states, where $Q$ rows are present. From Eq. (43) the horizontal phases must satisfy a telescope sum

$$
\sum_{j=j_{1}}^{j_{2}+1} \sin \overline{\phi_{0}^{x}}(j)=\overline{\psi_{0}}\left(j_{2}+1\right)-\overline{\psi_{0}}\left(j_{1}-1\right)=0,
$$

where we have used the fact that both rows $j_{2}+1$ and $j_{1}$ -1 must be either switched or in the boundary cells, and thus $\overline{\psi_{0}}=0$ from Eq. (46) or Eq. (37). Now, Eq. (45) can be solved with Eq. (48) to obtain: ${ }^{42}$

$$
\overline{\phi_{0}^{x}}\left(j+j_{1}-1\right)=2 \pi f\left(j-\frac{n+1}{2}\right)
$$

with $j=1, \ldots, n$. This gives the time-averaged phases of the horizontal junctions in the bulk of the $Q$ region. Then, from Eq. (43), the mesh current in the same region can be computed as

$$
\overline{\psi_{0}}\left(j+j_{1}-1\right)=\sum_{\ell=1}^{j} \sin \overline{\phi_{0}^{x}}(\ell)=\frac{\sin (\pi f j)}{\sin (\pi f)} \sin [\pi f(j-n)]
$$

for $j=1, \ldots, n-1$. This procedure allows us to solve for each $Q$ region in the array independently.

The remaining variables are easy to find. Recall that $\overline{\psi_{0}}$ vanishes everywhere in the $S$ region. The rest of the horizontal junctions $\overline{\phi_{0}^{x}}$ lie either between two $S$ rows, or between an $S$ row and a boundary cell. In either case, it follows from Eq. (43) that

$$
\overline{\phi_{0}^{x}}(j)=0, \quad \text { inside an } S \text { region. }
$$

Finally, the wave numbers $k(j)$ for the switched rows $(j$ $\in S)$ can be calculated from Eq. (44). One notices that $k(j)$ can change from a row to another, depending on the adjacent horizontal junctions $\overline{\phi_{0}^{x}}$. On the other hand, if there is an $S$ region with three or more rows, $k(j)=2 \pi f$ for all the rows except for the two rows at the top and bottom borders of the region; this is because $\overline{\phi_{0}^{x}}=0$ inside the region. In this sense, $k_{0}=2 \pi f$ is the "natural" wave number for $S$ rows.

This concludes the solution of the simplified equations (43)-(47). We now exemplify this procedure with four RS states of an array with $N^{y}=7$ rows, as depicted in Fig. 2. In Sec. IV we will compare the predictions with our numerics.

Pattern 1: $S=\{1, \ldots, 7\}$. This is the totally row-switched state in which there is no $Q$ region. Thus, the horizontal phases are

$$
\overline{\phi_{0}^{x}}=(0,0,0,0,0,0,0,0) .
$$

[The $j$ th component of the vector is $\overline{\phi_{0}^{x}}(j)$. Note this $j$ runs through $1, \ldots, 8$ for the seven-row array.] In addition, $\overline{\psi_{0}}(j)$ $=0$, and $k(j)=2 \pi f=k_{0}$ for all rows $j=1, \ldots, 7$.

Pattern 2: $S=\{2,4,6\}$, (and so, $Q=\{1,3,5,7\}$ ). In this symmetric pattern there are four $Q$ regions, each consisting of only one row, and three one-row $S$ regions. By solving each $Q$ region independently, we find

$$
\overline{\phi_{0}^{x}}=\pi f(-1,1,-1,1,-1,1,-1,1) .
$$


Then, for the $S$ rows $j=2,4,6$, we have $\overline{\psi_{0}}(j)=-\sin (\pi f)$ and $k(j)=4 \pi f$. That is, the three $S$ rows have an identical wave number but are different from the natural $k_{0}$.

Pattern 3: $S=\{4\}$. In this case the two symmetric $Q$ regions, rows 1-3 and 5-7, are separated by the central $S$ row. We obtain

$$
\overline{\phi_{0}^{x}}=\pi f(-3,-1,1,3,-3,-1,1,3) .
$$

The wave number of the $S$ row is $k(4)=8 \pi f$.

Pattern 4: $S=\{2,3,4,7\}$. In this highly asymmetric switching pattern there are two $Q$ regions. We obtain

$$
\overline{\phi_{0}^{x}}=\pi f(-1,1,0,0,-2,0,2,0) .
$$

The $S$ rows have the following wave numbers: $k(2)=3 \pi f$, $k(3)=2 \pi f$, and $k(4)=k(7)=4 \pi f$. Note that the rows 2-4 are contiguous but all have different wave numbers. The row 3 is surrounded by other $S$ rows, hence has the natural wave number. Meanwhile, the rows 2 and 4, which are contiguous to $Q$ regions have different wave numbers.

A similar bulk approximation can be obtained for the other limit of the aspect ratio. We present this small aspectratio case in the Appendix.

One might wonder what has happened to the phase constants $\delta(j)$ of the switched rows (42). Indeed, the $\delta(j)$ have disappeared in the simplified system (43)-(47), making them arbitrary. However, simulations show that the switched rows are weakly coupled, so that the $\delta$ 's drift to some particular values (if $f \neq 0$ ). This phase locking has been noticed in the completely switched state and left unexplained. ${ }^{6,8,10}$ As we show in the numerics of Sec. IV, it is also a feature of the partially RS states. The indeterminancy of $\delta$ in our analysis is not merely due to the assumption of the whirling solution (42). Rather, it is already inherent in the dc equations (29)(32) for which the addition of a constant to all the $\overline{\phi_{0}^{y}}(i)$ within any switched row leaves the system unchanged. Since the drift occurs in a much slower time scale than the basic oscillation frequencies, ${ }^{44}$ we conjecture that the $\delta(j)$ could be determined from solvability (or secularity) conditions that might arise from higher orders of the expansion. That was the case in one-dimensional series arrays ${ }^{40}$ where a similar slow phase drift and eventual locking was explained in that manner. However, it is beyond the scope of this paper to develop a similar calculation for the $2 \mathrm{D}$ array, and, in the following, we will use the values of $\delta(j)$ obtained from the numerical simulations.

\section{Solving the full dc equations}

We now consider how to solve the full dc system beyond the bulk approximation-a problem which requires, in general, numerical solution. It is important, however, to note that the decoupling of the equations introduced by the switched rows is still present so that the problem reduces to calculating static solutions of smaller arrays.

The important point to recall is given by Eq. (38): the mesh current is still zero in all $S$ rows. This breaks the array into disjoint $Q$ regions, as far as the leading-order dc part is concerned. Mathematically, this means that Eqs. (29), (30), and (32) are closed within each $Q$ region, and can be solved independently. This system is identical to the superconducting (static) equations for an isolated 2D array of the same size as the $Q$ region. When this subproblem of finding the static solutions for the independent $Q$ regions is solved, the remaining unknowns, $\overline{\phi_{0}^{y}}$ in the $S$ regions, can be determined from Eq. (30). This two-step procedure is completely analogous to the one used in the large aspect-ratio approximation, except that $\overline{\phi_{0}^{y}}$ in the $Q$ rows now depends on $i$, and, thus, $\overline{\phi_{0}^{y}}(i, j)$ in the $S$ rows cannot have the form given in Eq. (42).

How do we obtain the static configurations? Since a $Q$ region can take any size in the $j$ direction (up to $N^{y}$ ), we need, in short, a general calculation scheme of static states for an arbitrary rectangular array. An analytical formula is not known even for the no-vortex solutions (one of the many possible superconducting states) we are primarily concerned with. Thus, they must be found numerically. ${ }^{45,46} \mathrm{~A}$ rare exception is the ladder array, of size $N^{x} \times 1$, for which an accurate analytical approximation has been obtained. ${ }^{47}$ It shows that the full static solution differs from the bulk approximation in the existence of skin layers near the left and right edges. Crucially, resolving the phases in the skin layers is central to the existence and stability of the static solution. The ladder case is special but important since it is the most persistent in the parameter space among $Q$ regions of a given width. $^{22,46}$ In Sec. V we show how the stability of the RS patterns is connected with the stability of the static states.

\section{Analysis of the ac equations}

We now study the ac system (33)-(36). We only need to note that this is a linear system which is forced by the sinusoidal drive $\sin \left(\tau+\overline{\phi_{0}^{y}}\right)$. Therefore, if the dc solution is known, the ac system is simple to analyze.

Assuming that the homogeneous part decays, the solution locks to the forcing and the time dependence can be factored out as

$$
\left[\begin{array}{c}
\phi_{2}^{x} \\
\phi_{2}^{y} \\
\widetilde{\psi_{0}}
\end{array}\right](i, j, \tau)=\left[\begin{array}{l}
A \\
B \\
C
\end{array}\right](i, j) \exp (\tau \sqrt{-1})+\text { c.c. }
$$

where c.c. denotes complex conjugate. Then, the spatially dependent complex amplitudes must satisfy

$$
\begin{gathered}
-A(i, j)=C(i, j)-C(i, j-1), \\
-B(i, j)=-C(i, j)+C(i-1, j)+f(i, j), \\
A(i, j+1)-A(i, j)=B(i+1, j)-B(i, j)
\end{gathered}
$$

with

$$
f(i, j)=\left\{\begin{array}{cc}
\frac{\sqrt{-1}}{2} \exp \left[\overline{\phi_{0}^{y}}(i, j) \sqrt{-1}\right] & \text { if } j \in S \\
0 & \text { if } j \in Q .
\end{array}\right.
$$

Eliminating $A$ and $B$ from the equations, we obtain a discrete Poisson equation for $C$ : 


$$
\Delta C=-\mu
$$

with the source term

$$
\mu(i, j)=f(i, j)-f(i+1, j)
$$

and, from Eq. (37), boundary conditions

$$
C=0 \quad \text { in the boundary cells. }
$$

In the rectangular domain this problem can be solved via the double discrete Fourier-sine series

$$
C(i, j)=\sum_{m=1}^{N^{x}} \sum_{n=1}^{N^{y}} \hat{C}_{m, n} \sin \left(\frac{m i \pi}{N^{x}+1}\right) \sin \left(\frac{n j \pi}{N^{y}+1}\right)
$$

with

$$
\hat{C}_{m, n}=\frac{1}{a_{m, n}^{2}} \sum_{i=1}^{N^{x}} \sum_{j=1}^{N^{y}} \mu(i, j) \sin \left(\frac{i m \pi}{N^{x}+1}\right) \sin \left(\frac{j n \pi}{N^{y}+1}\right),
$$

where

$$
\begin{aligned}
a_{m, n}^{2}= & \left(N^{x}+1\right)\left(N^{y}+1\right) \\
& \times\left\{\sin ^{2}\left(\frac{m \pi}{2\left(N^{x}+1\right)}\right)+\sin ^{2}\left(\frac{n \pi}{2\left(N^{y}+1\right)}\right)\right\} .
\end{aligned}
$$

Finally, $A$ and $B$ are determined from Eqs. (52) and (53). This completes the analysis of the leading-order equations.

\section{NUMERICS}

\section{A. Finding RS states in simulations}

To test the validity of the analysis developed in the previous section we now compare its predictions with numerical results. The full governing equations (17) and (20), together with the boundary conditions (6), are integrated using the standard fourth- and fifth-order Runge-Kutta integrator with adaptive time step. Ours is an elementary nonoptimized version of the previous mesh-formulated code ${ }^{32,18}$ which enables us to switch between no-inductance and simple inductance models. The results presented here do not include inductances nor the effects of temperature and disorder.

Since most of the analysis has assumed the large aspectratio approximation, we study an array with $N^{x}=31$ and $N^{y}=7$, with small damping $\Gamma=0.2$ and a moderate external field $f=0.1$. We use as initial conditions the predicted large aspect-ratio dc approximations $\overline{\phi_{0}^{x, y}}$ (and the corresponding first-time derivatives). They are expected to be close enough to the true RS states to facilitate convergence, but we leave the ac part to be adjusted by the system. We choose a value for $I_{\mathrm{dc}}$ between 0 and 1 , and monitor whether the ensuing dynamical state is indeed the attempted RS pattern.

The system, of course, does not always converge to the row-switched state we have targeted; the chosen initial condition may be out of the basin of attraction of the target state, or the state may not exist, or it may be unstable for the chosen parameters. The outcome from using "wrong" parameters is, as far as we have tested, as follows: If $I_{\mathrm{dc}}$ is too large, then vortices start to enter in some of the rows we have

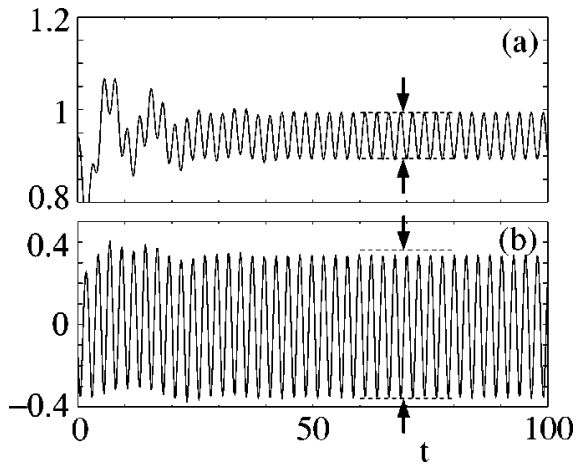

FIG. 3. Time evolution in the central (switched) row of pattern 3: (a) horizontal phase $\phi^{x}(16,4, t)$ and (b) mesh current $\psi(16,4, t)$. After a short transient, the solution converges onto a periodic attractor with dc values and ac amplitudes (shown together as the bands delimited by the dotted lines) well predicted by the analytical formulas in Sec. III.

initially set quiescent; if $I_{\mathrm{dc}}$ is too small, then the rows we have set switched cannot maintain the whirling motion, and exhibit retrapping, become quasiperiodic, or show highly nonlinear oscillations. In those cases, we then adjust $I_{\mathrm{dc}}$ until we find the clean periodic RS solutions which we aimed at.

Not only must we tune $I_{\mathrm{dc}}$, but the damping parameter $\Gamma$ must be small enough in order to find clean RS states. If $\Gamma$ is too large, it is difficult to find any partial RS states at all. For intermediate values, such as $\Gamma=0.4$, some RS patterns are observed, but some others cannot be found. For the underdamped case $\Gamma=0.2$ studied here, it becomes easy to find an appropriate range of $I_{\mathrm{dc}}$ in which the system converges to the expected RS pattern. This dependence on the damping is in qualitative agreement with experimental findings. ${ }^{17}$ It is also consistent with our assumption of the high-frequency limit since a smaller $\Gamma$ for a given $I_{\mathrm{dc}}$ corresponds to a larger $\omega$.

Generally, patterns with large quiescent regions are more difficult to obtain; for example, the RS state $S=\{1\}$ (with one $Q$ region of six rows) has a smaller interval of suitable $I_{\mathrm{dc}}$ than the symmetric pattern 3: $S=\{4\}$ (with two $Q$ regions of 3 rows), even though both states have only one $S$ row. These observations and the above parameter dependences will be discussed in more detail in Sec. V.

Before presenting detailed comparisons between numerics and analysis for patterns $1-4$, we first illustrate convergence in Fig. 3. There we show the time evolution of two variables in the array for pattern 3 , using $I_{\mathrm{dc}}=0.5$. Since the initial condition (taken as the bulk approximation) is not a solution of the full system, there is a short transient $(t<50)$ until the system settles onto a periodic attractor. Recall that only row 4 is switched in this pattern. Figure 3(a) shows the phase $\phi^{x}(16,4)$ of a horizontal junction adjacent to the switched row and in the middle of the row, where the large aspectratio (bulk) approximation is expected to be valid. The approximated average value is $3 \pi f \approx 0.94$, as predicted in Sec. III. Similarly, the mesh current in the central cell $\psi(16,4)$, shown in Fig. 3(b), is $\psi=0$ on average with some oscillations, as expected in any switched row. Not only the average values but the ac amplitudes are also well estimated from the ac leading-order equations, as demonstrated in Fig. 3. 


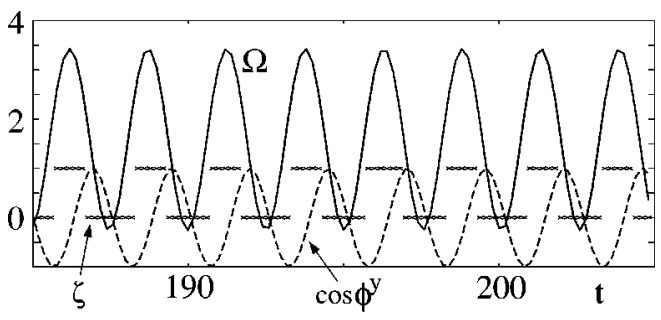

FIG. 4. Time evolution of the vorticities in the middle of the array of pattern 3 . The solid curve depicts the current vorticity $\Omega(16,4, t)$, while the topological vorticity $\zeta(16,4, t)$ switches discontinuously between 0 (no vortex) and 1 (one vortex in the cell). This discontinuous "tagging" of the position of the vortex is clarified by the dotted curve, which corresponds to $\cos \phi^{y}(16,4, t)$. Inspection of that magnitude indicates that every time it becomes -1 (i.e., the phase is equal to $\pi$ ) one topological vortex enters the cell (and $\zeta$ is increased by one).

\section{B. Vortex motion}

We illustrate now the two vorticities defined in Sec. II. In Fig. 4 we show the current vorticity $\Omega$ and the topological vorticity $\zeta$ both at the central cell $(15,4)$ of pattern 3 after convergence. They display similar periodic behavior though $\Omega$ is continuous whereas $\zeta$ switches discontinuously between 0 and 1 . $\zeta$ becomes unity when a charge enters the cell, which occurs in this case when $\phi^{y}(16,4)$, the left junction, crosses $\pi$ (modulo $2 \pi$ ). Therefore, $\zeta$ becomes unity when $\cos \phi^{y}(16,4)=-1$, as shown in the figure. Similarly, when the right junction $\phi^{y}(17,4)$ (not shown) turns and crosses $\pi$, the charge $\zeta$ is reset to zero.

As a complement to the time evolution of the vorticities in one cell, we also show snapshots of their spatial distributions for all patterns 1-4 in Figs. 2 and 5. In Fig. 5 each cell is shaded according to the value of the current vorticity $\Omega(i, j)$ : dark regions indicate positive large $\Omega$, while bright parts correspond to negative $\Omega$. The same snapshots, but showing the topological charges $\zeta$, are given in Fig. 2. Even though $\Omega$ represents the spatial structure more clearly, we observe that a charge in a cell corresponds to a peak of $\Omega$, and that the charges propagate through the array on top of the underlying wave.

Thus, we can use $\zeta$ to visualize the wavelength and the propagation speed in each row. In all patterns $1-4$, the charges move across the array at a nearly constant speed, as seen in the space-time plots of $\zeta$ in Fig. 6. They propagate only through the $S$ rows, and are apparently in phase in all rows for patterns 1 and 2. However, in pattern 4 the $S$ rows are not in phase, and the propagation velocities vary from row to row. Thus, the simplistic picture where vortices carry all the flux and move with the same speed in all the $S$ rows within a pattern ${ }^{32}$ leads to estimated speeds in disagreement with our simulations. This further proves that the underlying assumption that the topological vortices are particlelike objects which concentrate all the flux is not accurate. Instead, the RS solutions are not localized states and the flux is spatially distributed, as suggested by previous work ${ }^{2,10}$ and demonstrated in our analysis. Therefore, in these states, the topological vortices merely mark where the rotating junctions cross $\pi(\bmod 2 \pi)($ see Sec. II D), and they travel at the phase velocities of the underlying (nonlocalized) waves. Our

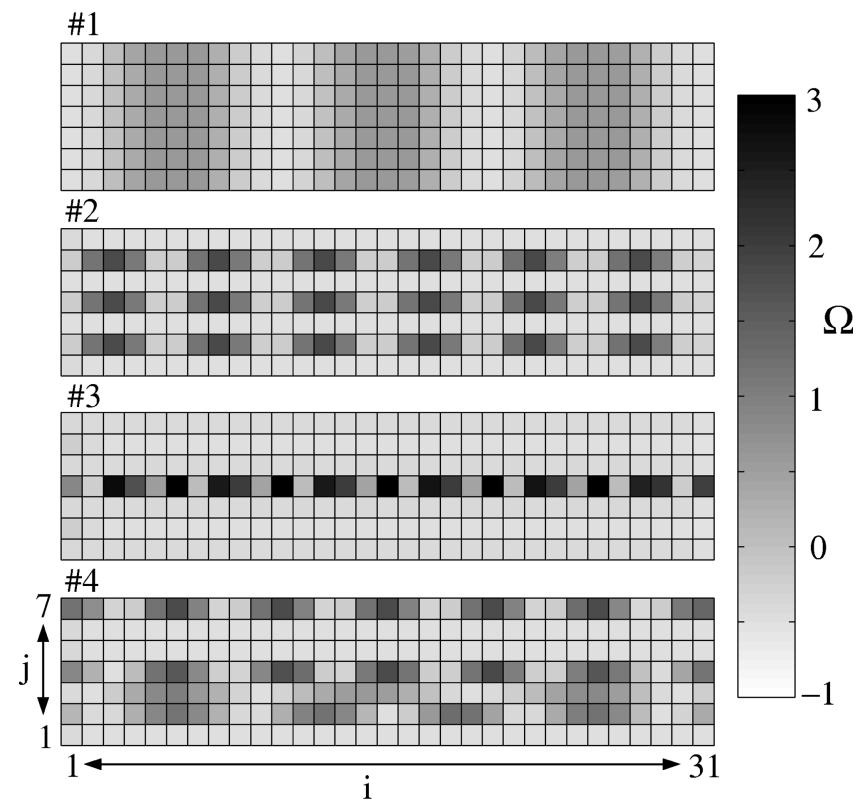

FIG. 5. Snapshots of patterns 1-4 showing spatial distributions of the current vorticity $\Omega$ as density plots. Dark regions correspond to large positive $\Omega$. Compare them with Fig. 2 where the same spatial patterns are shown in terms of the topological charge $\zeta$. We observe that the topological vortices are generally located on peaks of $\Omega$, and propagate locked to the underlying wave.

analysis correctly estimates the spatial wave numbers (thus, the propagation speeds) as shown below.

\section{Spatial structures after convergence}

We now present a quantitative comparison of the analysis of Sec. III to numerical simulations. The analytical predictions correspond to the large aspect-ratio (bulk) approximation both for the dc and the ac components. For the numerics, we simulate a $31 \times 7$ array, and the system is allowed to converge to periodic solutions for patterns $1-4$ using $\Gamma$ $=0.2, f=0.1$, and $I_{\mathrm{dc}}=0.6$ (except for pattern 3 , in which $I_{\mathrm{dc}}=0.5$ had to be used).

We first check the predicted spatial wave numbers $k(j)$ in the $S$ rows just discussed above. In Fig. 7 we show a "snapshot" of the $\phi^{y}$ in the $S$ rows $(2,3,4,7)$ of the nontrivial pattern 4 . To ease the display and comparison of the numerical results, we have juxtaposed the rows one after the other. Within each row, the spatial dependence is clearly linear, thus justifying the whirling mode assumption (42). The predicted wave numbers $k_{2}=3 \pi f, k_{3}=2 \pi f$, and $k_{4}=k_{7}$ $=4 \pi f$ (dashed lines) are almost indistinguishable from the numerics (solid lines) except for small deviations close to the edges.

Recall that in our analysis of the dc equations the interrow phase differences $\delta(j)$ are predicted to be arbitrary in Eq. (42). Hence, only the slope of the spatial dependence is known and the dashed lines are adjusted to match at the center of each row. Conversely, this is a way to determine the $\delta(j)$ from the numerical simulations. For the four patterns, we obtain: 


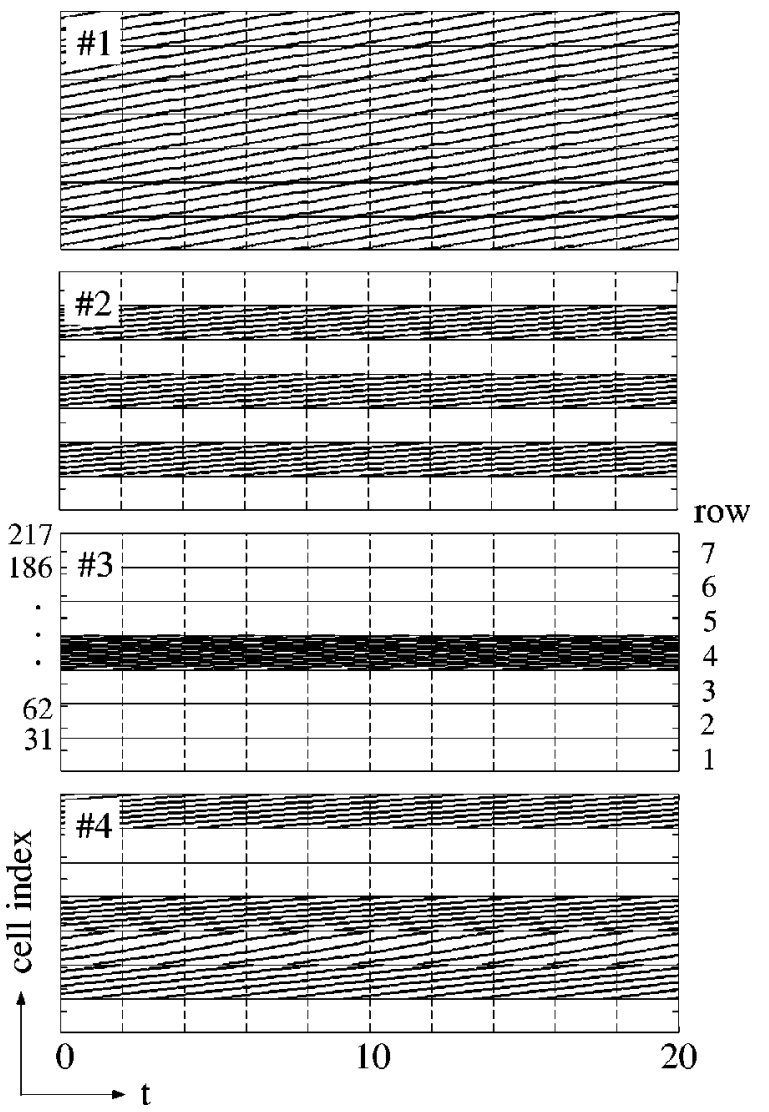

FIG. 6. Space-time plots of the propagation of topological vortices for patterns 1-4. The vertical (space) axis is the cell index: the cell $(i, j)$ is indexed one dimensionally by $i+N^{y}(j-1)$ by juxtaposing row after row. Within each of the symmetric patterns (1-3), the vortices in the switched rows have the same wavelength and are in phase. However, in the asymmetric pattern 4, the spatial wave numbers differ from row to row.

$$
\begin{aligned}
& \text { Pattern 1: } \delta(1)=\delta(7)=0, \delta(2)=\delta(6)=0.05, \\
& \delta(3)=\delta(4)=\delta(5)=0.1 . \\
& \text { Pattern 2: } \delta(2)=\delta(4)=\delta(6)=0 . \\
& \text { Pattern 3: } \delta(4)=0 . \\
& \text { Pattern 4: } \delta(2)=-1.8, \delta(3)=-4.7, \delta(4)=0.2, \\
& \delta(7)=0 .
\end{aligned}
$$

Note that in each case one $\delta(j)$ is set to zero and taken as the reference, which is equivalent to choosing the origin of $t$. In the following, we will use these numerical values of $\delta$ when needed (most importantly, for the analytical values of the ac components).

Next, we compare the predicted dc values with the numerical mean values after convergence. As we showed in Fig. 3(a), each horizontal junction $\phi^{x}$ librates around some dc value after convergence. These average values are plotted (solid lines) and compared to the large aspect-ratio approximation (dotted lines) in Fig. 8. The prediction is uniform within each row because edge effects were neglectedconsequently, it works well everywhere except close to the right and left ends. Similarly, the dc values of the vertical junctions in the $Q$ rows (not shown) are all predicted to be $\phi^{y}=\arcsin I_{\mathrm{dc}}$ in the bulk approximation, and the agreement is very good except for the edge corrections.

In Fig. 9 we show the ac amplitudes of the vertical junctions, i.e., the $|B(i, j)|$ calculated in Sec. III C, converted into

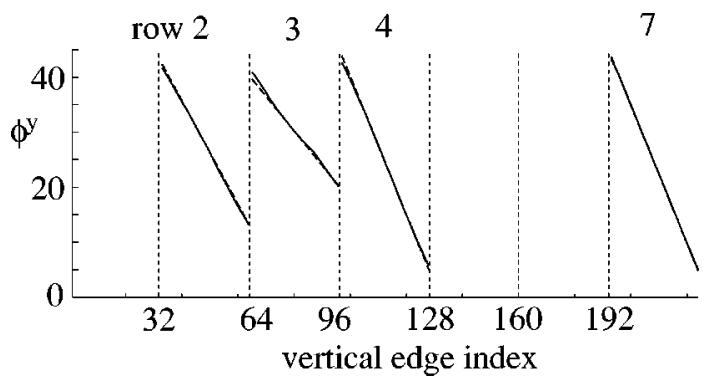

FIG. 7. A snapshot of the vertical phases $\phi^{y}$ in the switched rows $S=\{2,3,4,7\}$ of pattern 4 in a $N^{x}=31$ by $N^{y}=7$ array. Each solid line connects the numerical phases of the 32 junctions in each switched row. The dashed lines (almost overlapping with the solid ones) are the analytical approximation in Sec. III which predict the observed spatial wave number very well. The horizontal axis denotes the "vertical edge index," which numbers the vertical junctions consecutively as $i+\left(N^{x}+1\right)(j-1)$ for $i=1, \ldots, N^{x}+1$ and $j=1, \ldots, N^{y}$. This enables us to display the $2 \mathrm{D}$ array in a single axis by juxtaposing one row after the other. As a guide to the eye, vertical dotted lines are added to separate the rows.

voltages. As observed, symmetric patterns 1-3 have rather constant amplitudes throughout the middle of each row, but the asymmetric pattern 4 shows spatial fluctuations. Our estimates, shown as dotted lines, reproduce the spatial structure fairly well. It is quite remarkable that our approximation roughly captures the behavior at the right and left boundaries even though we have used the bulk approximation $\overline{\phi_{0}^{y}}$ [together with the numerical $\delta(j)]$ to solve the ac system.

Since the mesh current is determined from the phase configurations $\phi^{x, y}$, it also compares well with the large aspectratio approximation. Thus, we do not display the quantitative comparison of $\psi$, and instead present more descriptive 2D contour plots of the numerical $\psi$ on the $31 \times 7$ array geometry. The contour curves of the dc component of $\psi$ are shown in Fig. 10. If the 2D array were continuous, the induced currents would flow (on average) along these curves. Since the array is discrete, the flow is restricted to the branches, but the level curves still describe roughly the way the currents circulate. Furthermore, the dc values in the $S$ rows are nearly zero, as expected. In the $Q$ regions, currents circulate in the clockwise direction $\left(\overline{\psi_{0}}<0\right)$ on average. This would induce a magnetic field through the $Q$ regions in the opposite direction to the external field $f$. Although it is interesting to ask whether the induced field cancels the external one to produce a Meissner-like region, that question only makes sense when inductances are included. Note also that all contour plots are almost left-right symmetric, but show a slight asymmetry. This is presumably due to the presence of edges and the preferred direction $(\hat{\mathbf{x}})$ of propagation of the waves across the $S$ rows. Such details are not captured by the bulk approximation and the full solution of the dc equations becomes necessary.

Finally, the amplitudes of the ac oscillations of $\psi$ are shown in another set of contour plots in Fig. 11. The observed nodal structures (typical in linear forced systems in a bounded domain) show the spatial distribution of the modes locked to the driving dc solution. The magnitude of these ac amplitudes is comparable to the dc values in Fig. 10, even though the oscillating components of the phases $\phi$ are much 

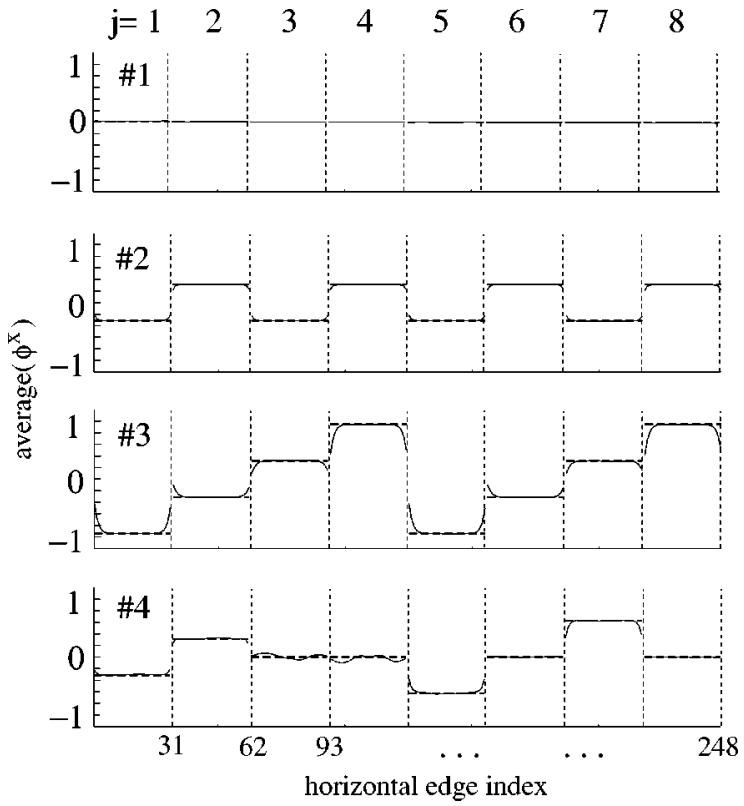

FIG. 8. The dc values of $\phi^{x}$ for patterns $1-4$, showing the spatial distribution of the average horizontal phases. The horizontal axis is the "horizontal edge index," defined as $i+N^{x}(j-1)$ for $\phi^{x}(i, j)$. There are $N^{y}+1=8$ horizontal edges so that $j$ runs from 1 to 8 . For each $j$, the $N^{x}=31$ phases in the same row are connected. The dotted lines are from the large aspect-ratio approximation which accurately estimates the numerical results in the bulk of the array. The dc values are predicted to be multiples of $\pi f$. The approximation neglects the effects of the left and right edges, and, thus, inevitably misses the skin layers at both lateral boundaries. Vertical dashed lines mark the separation between $j$ 's.

smaller, of $O\left(\omega^{-2}\right)$, than their dc values. This is consistent with our analysis which assumes that the mesh current has dc and ac components both of $O(1)$.

\section{PARAMETER REGION FOR RS STATES}

In this section we determine where in the parameter space we expect RS solutions. This is a difficult task, partly because the parameter space is large. Even after neglecting induced fields (i.e., $\lambda_{\perp}=\infty$ ) we are left with three parameters: $f, \Gamma$, and $I_{\mathrm{dc}}$. In addition, there can be multiple attractors coexisting for a given parameter set. Recall, for example, how in the previous section patterns 1,2 , and 4 were obtained using an identical parameter set, and pattern 3 also used a similar $I_{\mathrm{dc}}$ value. A thorough determination of the parameter regime would then require a rigorous study of the bifurcations of the branches of all the attractors - an exploration which exceeds the scope of this article, and is perhaps too detailed to justify the necessary effort. Here, we take a more heuristic approach, and make several assumptions to estimate the current interval $\left[I^{\mathrm{min}}, I^{\mathrm{max}}\right]$ in which a given RS state is an attractor, as a function of $f$ and $\Gamma$. We base our assumptions on the results of previous sections, and we demonstrate their validity by additional calculations in the following.

\section{A. Upper current limit}

We first estimate the upper current $I^{\max }$ at which a given RS state ceases to be an attractor. Our first assumption states
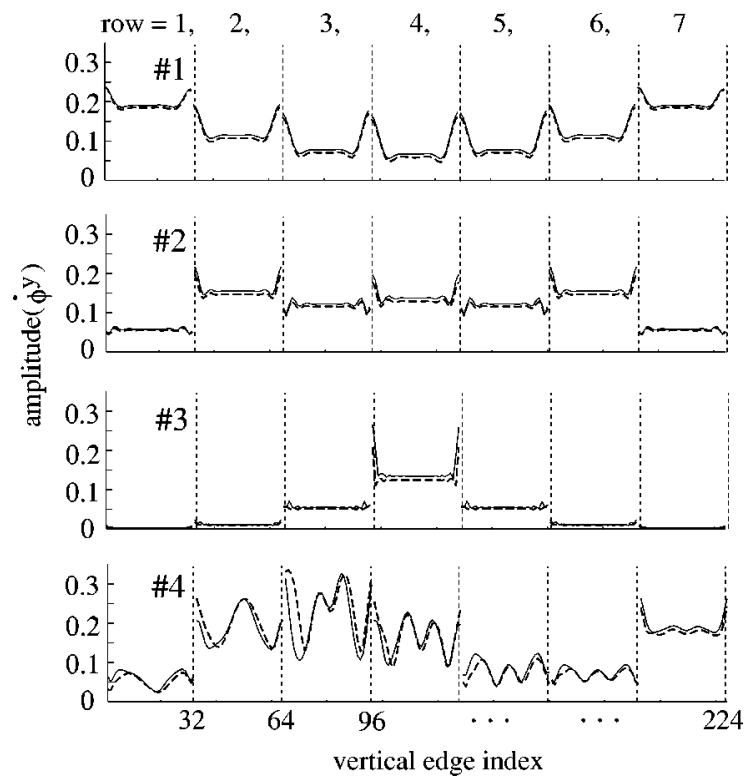

FIG. 9. Dimensionless ac voltage amplitudes $\dot{\phi}^{y}$ for patterns $1-4$, plotted against the vertical edge index, defined in Fig. 7. For each row its $N^{x}+1=32$ points are connected. The large aspect-ratio approximation, shown as dashed curves, can describe the spatial distribution fairly well.

that this upper limit is reached when vortices enter any of the $Q$ regions from the edge. The entrance of flux might produce further switching of rows (resulting in another RS state where the original $Q$ region has been subdivided), or a more complicated state where the flux remains static or moves through the original $Q$ region in a highly nonlinear motion. In either case, the original RS state is no longer maintained. As discussed in Sec. III, each $Q$ region is decoupled up to the dc leading order and is equivalent to an isolated superconducting array of the same dimensions. If, as we assume, no vortex has been trapped beforehand in the $Q$ regions, arrays with more rows depin at smaller values of $I_{\mathrm{dc}}$, as can be shown numerically. ${ }^{46}$ Therefore, our second assumption is that, as $I_{\mathrm{dc}}$ is raised in an RS state, a vortex first enters the largest of the remaining $Q$ regions, causing further breakup of the array.

Thus, if the depinning current for the no-vortex superconducting state of any number of rows is known, these two assumptions enable us to estimate $I^{\text {max }}$ for any given pattern. For example, pattern $3(S=\{4\})$ has two $Q$ regions of the same size (three rows). We expect then that this state is not sustainable beyond the depinning current of a $31 \times 3$ array. At zero temperature and without disorder, the likely scenario is that flux enters the center row of each of the two regions, so that a new RS state, pattern $2(S=\{2,4,6\})$, ensues. This state has now four $Q$ regions, each consisting of one row. The upper $I_{\mathrm{dc}}$ value for this state should coincide with the depinning current of the $31 \times 1$ "'ladder' array. Beyond this value all rows switch and pattern 1 is obtained. We have indeed observed such a sequence of row-switching events when we gradually increase $I_{\mathrm{dc}}$ from zero, using a clean initial condition: $\phi=\dot{\phi}=0$ everywhere. Similarly, the largest $Q$ region in pattern 4 has 2 rows. Therefore, $I_{\mathrm{dc}}$ should coincide with the depinning current of a superconducting novortex $31 \times 2$ array. In Table I we summarize the excellent 


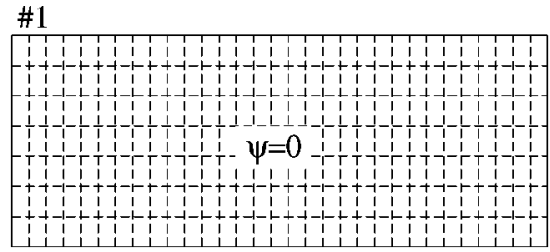

\#2

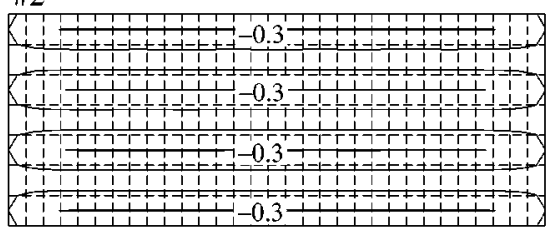

\#3

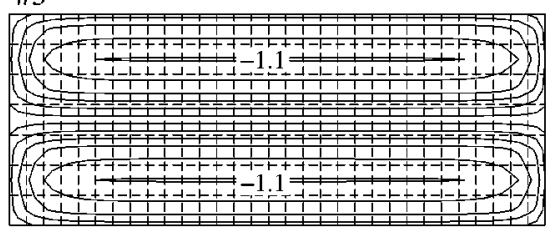

\#4

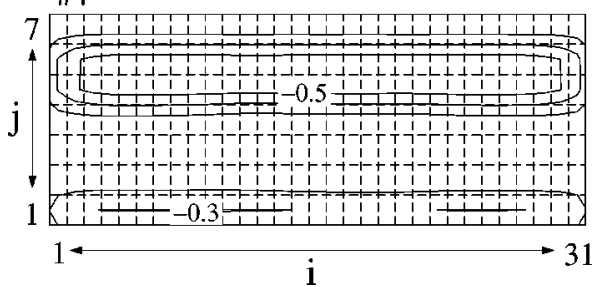

FIG. 10. Level curves of the dc mesh current $\psi$ for patterns 1-4, indicating how the induced circulating currents flow. The total current flow is the superposition of the induced flow and the injected uniform current flow. Contour levels at $-0.1,-0.3, \ldots,-1.1$ are drawn on the 2D grid of the $N^{x}=31$ by $N^{y}=7$ array. Pattern 1 shows little deviation from the uniform current flow on average, thus $\psi=0$ and no curves appear. In the other patterns, the dc values of $\psi$ in the switched rows are zero, while the values are negative in the quiescent rows. Therefore, currents circulate in the clockwise direction in each quiescent region "along" the level curves shown. Strictly, the currents are restricted to the grid, but the level curves provide an intuitive description of the flow. Note that the boundary condition $\psi=0$ is imposed at a half cell outside of the array borders; this explains why some of the contour curves intersect the array edges.

quantitative agreement between the numerically observed $I^{\max }$ values of several RS patterns, and the depinning currents of superconducting arrays with the same dimensions as their largest $Q$ region.

We have also tested our assumptions with four additional patterns, all with only one switched row: $S=\{4\}$ (the symmetric pattern 3), $S=\{3\}, S=\{2\}$, and $S=\{1\}$ (the most asymmetric pattern). This illustrates the dependence of the upper $I^{\max }$ not on the number of switched rows, as above, but on their location. For given $f$ and $\Gamma, I^{\mathrm{max}}$ becomes smaller as the switched row is shifted from the middle of the array to the bottom because the largest $Q$ region increases its size from $31 \times 3$ to $31 \times 6$. Excellent agreement is again obtained between our criterion and the numerical observations (Table I).

We now make the third assumption that enables us to obtain analytical estimates of $I^{\max }$ in some cases. We pro-

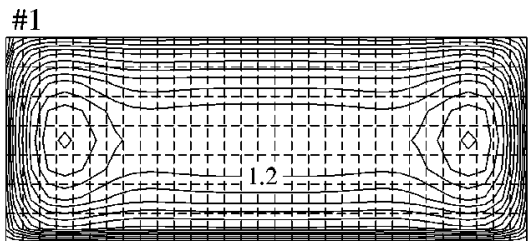

\#2

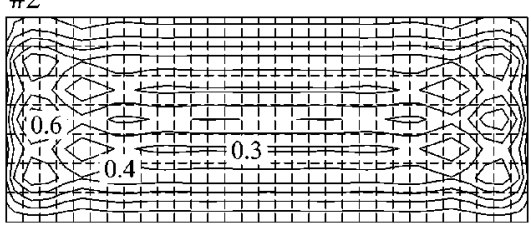

\#3

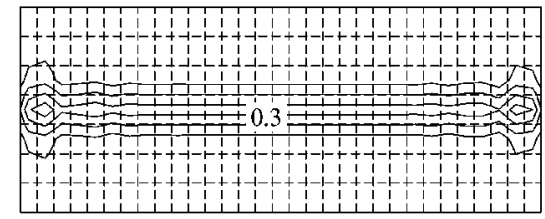

\#4

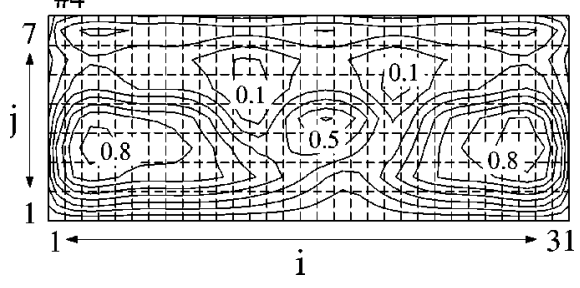

FIG. 11. Level curves of the ac amplitudes of the mesh current $\psi$ for patterns 1 (top) to 4 (bottom), on the $2 \mathrm{D}$ grid of $31 \times 7$ cells. Contour levels at $0.1,0.2, \ldots, 1.5$ are shown. The magnitudes are generally large in the switched rows, but even quiescent rows have some oscillations and, thus, are not purely superconducting. Our leading-order analysis in Sec. III C predicts that these ac oscillations obey the discrete Poisson equation with forcing originating from the dc components. The figure shows nodal structures typical in solutions to such a problem.

pose that, as the drive increases, just before the entrance of a vortex into a $Q$ region, a junction barely holds itself at a critical angle

$$
\phi^{\mathrm{crit}}= \pm \pi / 2 .
$$

When it is forced to turn beyond that value, depinning takes place, just as it would if the junction were uncoupled. Recall that the single uncoupled junction under an increasing drive becomes unstable through a saddle-node bifurcation at $I_{\mathrm{dc}}$ $=1$, with $\phi=\pi / 2$ as the bifurcation angle. Although the criterion for global depinning is different in a coupled array, this simple heuristic criterion has been used to predict the depinning current in ladder arrays with remarkable accuracy. $47^{4}$

Take, for instance, an array at zero temperature with small $N^{y}$ in a ground state with no pretrapped vortices. Then, the first junction to cross $\phi^{\text {crit }}= \pm \pi / 2$ is, for $f>0$ and $I_{\mathrm{dc}}>0$, the vertical junction which sits in the center row at the left edge. Thus, the flux would penetrate the array through that junction and destroy the RS state. This is readily deduced from the circulating current shown in Sec. IV which reinforces the drive near the left boundary. Such a current is due to the presence of the left and right boundaries, which our large aspect-ratio approximation neglected. A full analysis of 
TABLE I. Stability intervals $\left[I^{\mathrm{min}}, I^{\mathrm{max}}\right]$ (two middle columns) for eight RS patterns (two of them identical) in the $31 \times 7$ array using $f=0.05$ and $\Gamma=0.2$. The set $S$ denotes the switched row numbers, and patterns from Fig. 2 are labeled. The intervals are calculated numerically by gradually changing $I_{\mathrm{dc}}$ and following the corresponding branch of the RS state until instabilities appear. For example, pattern 2 is found in the interval $[0.335,0.945]$, for this set of parameters $(f=0.05, \Gamma=0.2)$. The upper limit $I^{\text {max }}$ can be predicted accurately by the depinning current $I^{\text {dep }}$ of the largest $Q$ region of each pattern (with dimensions $N^{x} \times N^{y}$, shown in parentheses). The lower limit $I^{\mathrm{min}}$ is harder to estimate, but the retrapping current $I^{\mathrm{ret}}$ of a single junction serves as a rough estimate: for $\Gamma=0.2$, the value is $I^{\text {ret }}=0.252$, which is smaller than the observed $I^{\mathrm{min}}=0.305-0.335$. The first four rows show patterns 1-4 from Fig. 2. The next four patterns all have a single $S$ row, but its location is different. Among these four, pattern 3 has the widest stability interval because its largest $Q$ region $(31 \times 3)$ has the smallest number of rows.

\begin{tabular}{lccccc}
\hline \hline$S$ & (Fig. 2) & $I^{\min }$ & $I^{\max }$ & $I^{\text {dep }}$ & $\left(N^{x} \times N^{y}\right)$ \\
\hline$\{1, \ldots, 7\}$ & $(1)$ & 0.335 & & & \\
$\{2,4,6\}$ & $(2)$ & 0.335 & 0.945 & 0.947 & $(31 \times 1)$ \\
$\{4\}$ & $(3)$ & 0.315 & 0.815 & 0.825 & $(31 \times 3)$ \\
$\{2,3,4,7\}$ & $(4)$ & 0.328 & 0.912 & 0.912 & $(31 \times 2)$ \\
$\{1\}$ & & & & \\
$\{2\}$ & & 0.305 & 0.625 & 0.622 & $(31 \times 6)$ \\
$\{3\}$ & & 0.305 & 0.685 & 0.681 & $(31 \times 5)$ \\
$\{4\}$ & $(3)$ & 0.315 & 0.776 & 0.778 & $(31 \times 4)$ \\
\hline \hline
\end{tabular}

the skin layers would be needed for a general analytical prediction, but there are two tractable limiting cases of interest.

The first case is a "small aspect-ratio" superconducting region, i.e., with many more rows than columns $\left(N^{y} \gg N^{x}\right)$. As discussed in the Appendix, a bulk approximation can then be used, which approximates accurately the phases near the left and right edges - because, in this case, the skin layers are located near the top and bottom boundaries. Should such a region be present in a $\mathrm{RS}$ state as a $Q$ region, it would be easily broken even with a small value of $I_{\mathrm{dc}}$. Since the central leftmost vertical junction has the largest angle (A4), it crosses the critical angle (62) at a critical current

$$
I_{\mathrm{SAR}}^{\max }=\frac{1}{2\left(N^{x}+1\right)}\left\{1+\frac{\sin \left[\pi f\left(2 N^{x}+1\right)\right]}{\sin (\pi f)}\right\} .
$$

From Eq. (63), the region remains stationary when $I_{\mathrm{dc}}$ $<I_{\mathrm{SAR}}^{\max }$ and $f<1 /\left(2 N^{x}\right)$. If $f>1 /\left(2 N^{x}\right)$, a vortex enters the $N^{y} \gg N^{x}$ region for any $I_{\mathrm{dc}}>0$. We have tested these conclusions numerically with good agreement. Moreover, note that other physical arguments ${ }^{37}$ predict that the edge barrier for the penetration of flux in this limit would be roughly given by $f_{\mathrm{c}} \sim 1 /\left(\pi N^{x}\right)$. The condition (63) results from the instability of a static state, and it does not depend on $\Gamma$, the damping coefficient.

The second case is the "ladder array," with $N^{x}$ columns and a single row. ${ }^{48}$ Its superconducting states, including states with trapped vortices, and their bifurcations have been studied comprehensively. ${ }^{47}$ One of the results of that work is the curve of the depinning current as a function of $f$, shown as a solid line in Figs. 12(a) and 12(b). This monotonically decreasing curve is again independent of $\Gamma$, and becomes insensitive to $N^{x}$, as soon as $N^{x}$ is greater than about 5. For $f$ up to about 0.46 , the depinning is caused by the disappearance of the no-vortex solution. This part of the curve is well approximated by the solution of the following implicit nonlinear equation ${ }^{47}$ which comes from imposing Eq. (62) to the leftmost junction:

$$
\arcsin \left(1-I_{\mathrm{LAD}}^{\max }\right)+\frac{r-1}{2 r} \arccos \left(I_{\mathrm{LAD}}^{\max }\right)=\pi f
$$

with $r=\alpha+\sqrt{\alpha^{2}-1}$ and $\alpha=1+\sqrt{1-I_{\mathrm{LAD}}^{\max 2}} / \cos \pi f$. After a crossover at $f \sim 0.46$, the static checkerboard pattern becomes more robust, and this formula ceases to be valid. We will not discuss RS states in this high range of $f$. If our assumptions are correct, this critical curve should predict the $I^{\max }$ of pattern 2 . In addition to the single comparison presented in Table I for this pattern, we test it for $\Gamma=0.2$ and 0.4 and several values of $f$ in Fig. 12. As shown there, the numerical $I^{\max }$ values of pattern 2 from simulations are predicted very accurately by the analysis of the depinning point of the ladder.

Up to now, we have assumed that the magnetic flux penetrates the $Q$ regions from the left edge of the array. However, the flux can also enter the array from the top or bottom boundaries of a $Q$ region in certain situations. Consider a $Q$ region with a large aspect ratio and no trapped vortices, but when the number of rows $N^{y}$ is large. In this case, the bulk approximation obtained in Sec. III B 1 can still be used. From Eq. (49) the maximum angle for the horizontal junctions is $\phi^{x}=N^{y} \pi f$ attained at the top and bottom edges of the region. It is clear that this value becomes larger than the critical angle (62) when $f>1 /\left(2 N^{y}\right)$. Thus, for a fixed $N^{y}$ while $f$ is increased, the flux would enter the $Q$ region roughly above that value of the frustration. The entrance of flux in this manner puts a limit on the applicability of our analysis. The assumed no-vortex $Q$ region is expected to exist only when the number of rows is smaller than about $1 /(2 f)$. Thus, our analysis does not apply for the initial 
(a) $\Gamma=0.4$

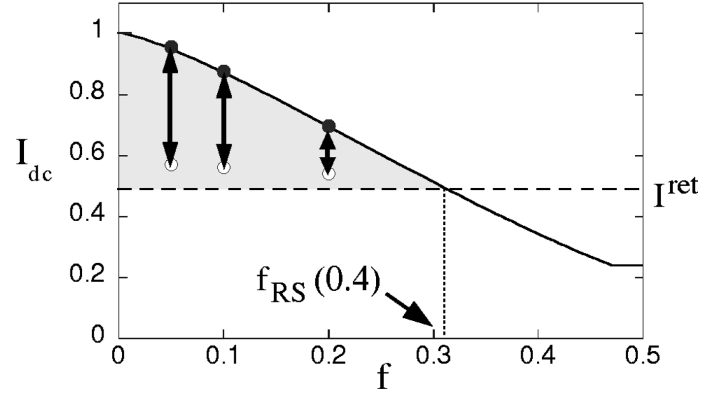

(b) $\Gamma=0.2$

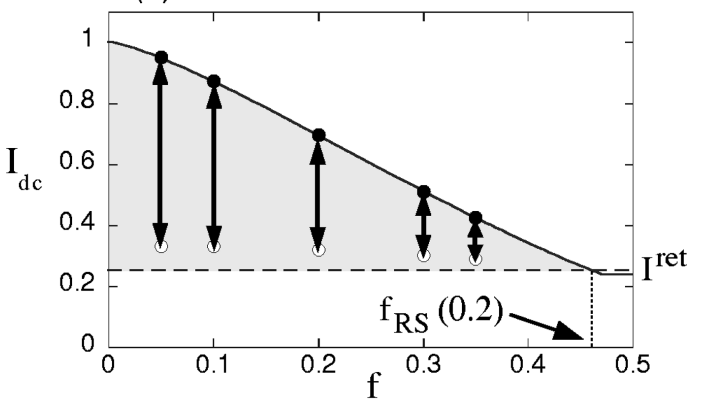

(c)

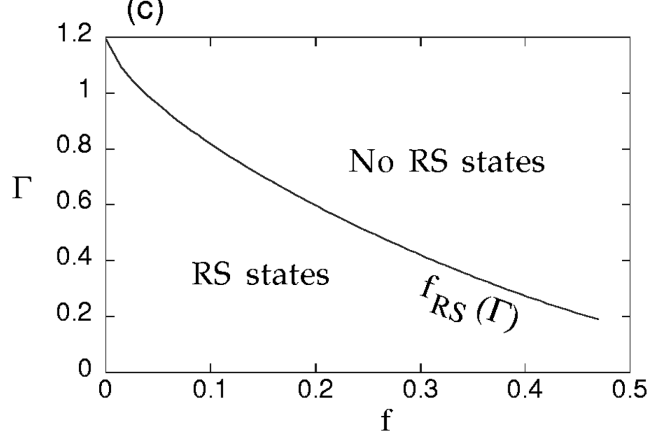

FIG. 12. (a) Stability region for pattern 2 with $\Gamma=0.4$. For $f=0.05,0.1,0.2$, we sweep $I_{\mathrm{dc}}$ to determine numerically the stability interval $\left[I^{\min }, I^{\max }\right]$ denoted by the vertical arrows with endpoints at $I^{\mathrm{min}}(\bigcirc)$ and $I^{\max }(\mathbf{O})$. The solid line is our estimate for $I^{\text {max }}(f)$, given by the depinning current $I^{\text {dep }}$ of a ladder array. The dashed line is an estimate for $I^{\mathrm{min}}$, given by the retrapping current $I^{\text {ret }}$ of the single junction at $\Gamma=0.4$. Therefore, the shaded section is the estimated region of the $I_{\mathrm{dc}}-f$ plane where pattern 2 exists, for $\Gamma=0.4$. Note that the region does not extend beyond a critical $f$ $=f_{\mathrm{RS}}(0.4)$. (b) Same as (a) but for $\Gamma=0.2$. Although the upper estimate $I^{\text {dep }}$ is unchanged, the lower estimate $I^{\text {ret }}$ decreases with $\Gamma$. Consequently, pattern 2 is expected to be observed in a larger parameter region for smaller $\Gamma$, as shown by the five intervals (arrows) obtained numerically. The region does not extend for $f$ larger than $f_{\mathrm{RS}}(0.2)$. (c) Phase diagram for the existence of RS states in the $f$ - $\Gamma$ parameter plane. The curve $f=f_{\mathrm{RS}}(\Gamma)$ separates the regions in which RS states may or may not appear. For $\Gamma \geqslant \Gamma^{*} \approx 1.2, I^{\text {ret }}$ $=1$, thus, no RS states are expected for any $f$. This diagram explains the previous (qualitative) observation that RS states occur most easily when junctions are underdamped and the applied magnetic field is small.

stages of the row-switching cascade in large arrays, when there are still $Q$ regions with many rows. However, even in such arrays, later steps of the cascade (when the $Q$ regions have been subdivided) can be described by assuming novortex $Q$ regions. In addition, our preliminary simulations indicate that keeping a vortex trapped in a $Q$ region becomes more difficult both in the presence of $I_{\mathrm{dc}}$ (which tends to expel the fluxoids from the $Q$ regions), and of self-fields (which tend to shield the $Q$ regions from the entrance of vortices).

\section{B. Lower current limit}

As shown in experiments and simulations, RS states appear only when the junctions are underdamped. However, all the critical currents calculated until now are independent of $\Gamma$. We claim that the explanation of the $\Gamma$ dependence of the RS states requires an estimate of the lower limit $I^{\mathrm{min}}$. Unlike the upper limit, in which the superconducting solution of a $Q$ region ceases to exist, our numerical observations suggest that the lower limit is caused by an instability mechanism in an $S$ region. As the bias current is decreased from the values in which a clean periodic RS state is observed, $S$ regions start to have trouble in maintaining fast whirling oscillations. Typically, the system begins to show amplitude modulations in a slow time scale, becomes highly nonlinear, or gets retrapped altogether.

The variety of possible scenarios makes an accurate estimate much harder than for $I^{\max }$. In order to make progress, we have to rely on a rather rough estimate, based on the dynamics of a single junction. Recall that the vertical junctions in an $S$ row are in the resistive (whirling) state. For a single underdamped junction, its inertia is enough to maintain a whirling solution until very close to the retrapping current $I^{\text {ret }}$, when it jumps back to the stationary state. Only near that value does a strong nonlinearity come into play. Ignoring the interjunction coupling, we use this current as our estimate for the lower limit $I^{\mathrm{min}}$ of an RS state. Because of collective effects, the state may not be immediately retrapped into a stationary state, or, on the contrary, be retrapped earlier. However, we expect that, as $I_{\mathrm{dc}}$ is lowered toward the $I^{\text {ret }}$ value, some nonlinear effects start to become apparent, so that the simple periodic RS state is altered.

The estimate of $I^{\text {ret }}$ is standard. ${ }^{36}$ For the underdamped case, (i.e., $\Gamma<\Gamma^{*} \approx 1.2$ ), the retrapping is produced through a homoclinic bifurcation at $I^{\mathrm{ret}}<1$, and the $I-V$ of the single junction is hysteretic. For all $\Gamma>\Gamma^{*}, I^{\mathrm{ret}}=1$, and there is no hysteresis. In general, $I^{\text {ret }}$ is calculated numerically, but an asymptotic expression, $I^{\mathrm{ret}} \sim 4 \Gamma / \pi$ can be used as $\Gamma \rightarrow 0$.

From the definition of $I^{\text {ret }}$, our estimate for $I^{\mathrm{min}}$ is thus independent of $f, N^{x}, N^{y}$, and the particular RS pattern, but depends on the damping $\Gamma$. The estimates for $\Gamma=0.2$ and 0.4 are shown as dashed lines in Figs. 12(a) and 12(b), respectively. The comparison with the numerical values of $I^{\mathrm{min}}$ (the point when the RS states lose their whirling character) is not so good, as expected. However, our estimate seems to serve as a reasonable first guess.

\section{C. $f$ - $\Gamma$ parameter region for $\mathrm{RS}$ states}

In the usual experimental setup, the $I-V$ characteristic of an array is measured by sweeping the dc current under a constant applied magnetic field at a fixed temperature (which controls the penetration depth $\lambda_{\perp}$ and damping $\Gamma$ ). For some combinations of the experimental variables (magnetic field and temperature) and, thus, of the underlying parameters $f$, $\Gamma$, and $\lambda_{\perp}$, the $I-V$ shows RS steps. For others, it does not. 
We will now summarize the preceding sections and combine their results to estimate the $(\Gamma, f)$ parameter region, in the limit $\lambda_{\perp}=\infty$, in which RS states appear.

First, in Sec. V A we showed two limiting cases in which $I^{\max }$ can be obtained analytically, i.e., when $N^{y}=1$ (ladder) and when $N^{y} \gg N^{x}$. Numerical simulations ${ }^{22,47,46}$ show that $I^{\max }$ changes monotonically between these two limits, as $N^{y}$ is varied. (This result is also expected from physical grounds: for fixed $N^{x}$, the magnetic flux penetrates the array more easily as $N^{y}$ is increased.) An obvious consequence of this is that the ladder array has the largest parameter domain for the no-vortex superconducting state. Therefore, recalling our link between depinning and row-switching, RS states whose $Q$ regions are all ladders, e.g., pattern 2, are thought to be the most stable in the same sense. In other words, when an isolated ladder of length $N^{x}$ cannot maintain superconductivity, the 2D array of size $N^{x} \times N^{y}$ cannot show rowswitched behavior. Consequently, the solid curve in Figs. 12(a) and 12(b) not only gives the upper limit $I^{\max }$ for pattern 2 , but also establishes the critical $I_{\mathrm{dc}}$, for each $f$, above which no RS states can be observed.

Second, we concluded in Sec. V B that the $I^{\mathrm{min}}$ of all RS states with damping $\Gamma$ can be estimated by the retrapping current of a single junction with the same $\Gamma$. These are the dashed straight lines in Figs. 12(a),12(b).

Hence, the RS states can only exist in the region contained between these upper and lower limits, shown as the shaded area in Fig. 12(a). Those limits intersect at a value $f_{\mathrm{RS}}(\Gamma)$ beyond which no RS state is possible. [See Figs. 12(a) and 12(b) for the procedure.] For junctions of moderate to large damping $\left(\Gamma>\Gamma^{*} \approx 1.2\right)$, the dashed line is above the curve, meaning that RS states are impossible for any $f$. On the other hand, for highly underdamped arrays $(\Gamma<0.2)$, the line always remains below the curve; hence, RS states are possible for any $f$ (although the region of $f$ near $1 / 2$ would need more careful consideration). Between these two extremes of damping, the line intersects the curve at the critical value $f_{\mathrm{RS}}(\Gamma)$, which constitutes a phase boundary in the $f-\Gamma$ plane. In other words, the parameter plane is divided into two regions (RS and no-RS) by the curve $f_{\mathrm{RS}}(\Gamma)$ in Fig. 12(c). This is in qualitative agreement with previous observations, and awaits more systematic experimental testing.

\section{SUMMARY AND OPEN PROBLEMS}

In this article we have used a weakly nonlinear perturbative analysis to study the row-switching phenomenon and to approximate the RS solutions. For the bulk of the array, we have obtained analytical expressions for the phase and current variables. In addition, we have estimated the parameter regime for their existence. For this, the consideration of the lateral edges has played an important role. The predicted spatial current distributions and the parameter regime could serve as a guide for more systematic experimental studies. In the rest of this section we briefly state open problems and possible future directions.

The leading-order solutions show good agreement with the numerics, but leave one phase per row undetermined. This is $\delta(j)$ in the large aspect-ratio approximation (42) and such an arbitrary phase is still present in the unapproximated leading-order dc equations, as discussed in Sec. III. How- ever, the full numerics show that there is a slow drift towards a specific set of $\delta(j)$ for each pattern. Several authors ${ }^{7,8,10}$ have studied this interrow phase locking in pattern 1, but a satisfying answer has yet to be developed. The zero-field limit $(f=0)$ is an exception in that exact neutral stability and a family of periodic solutions can be found, ${ }^{6,33}$ implying that there is no interrow locking. On the other hand, a slow drift starts to occur as $f$ is perturbed away from zero. ${ }^{44}$ We conjecture that the arbitrary phases should be constrained by a solvability condition in the higher-order expansions of our analysis, which is automatically satisfied when $f=0$. Finding that condition, however, is likely to be an elaborate task.

Our analysis is based on such simplifications as zero temperature, no disorder, and no self-fields. Clearly, the effect of relaxing these assumptions should be also investigated. Thermal noise, self-fields, and inhomogeneities alter the switching sequence in simulations of the row-switching cascade. $^{22,32,18}$ This might explain the irregularity of the rowswitching order observed experimentally by Trías $^{18}$ and Lachenmann $e t a l .{ }^{20}$ On the other hand, the directed use of disorder (e.g., by removing some of the edges in the array) might prove a valuable strategy to enhance the locking property of the arrays. ${ }^{49}$ Including inductances would also change the current distributions. ${ }^{4,31,32}$ Previous work, ${ }^{32,18}$ and our own preliminary calculations including self-inductances, show that RS states persist at least for small inductances. Our expansion could be extended to include inductances and then proceed to describe the modified solutions. However, qualitatively new phenomena can also arise. For example, it is known $^{18,19,26}$ that, if any inductance is included in the model, a coherent state (dynamical checkerboard pattern) emerges near $f=1 / 2$ when the RS states cease to exist.

In this article, we have only considered "clean" RS states, formed by whirling and no-vortex superconducting regions. Thus, we have assumed that the $Q$ rows do not contain any static vortices. It is generally expected that the depinning of a $Q$ region would become easier when it contains a pretrapped vortex. Therefore, the existence of states with static vortices probably does not affect the critical curve in Fig. 12(c). However, the question of how the depinning of a static 2D array depends on various parameters $\left(\Gamma, f, N^{x}\right.$, and $N^{y}$ ) is not fully understood, except in the case of the ladder, ${ }^{47}$ and requires further scrutiny. Similarly, the $S$ rows in the RS states were assumed to be in the whirling (normal resistive) state. Our simulations sometimes show "generalized" RS states which contain one or more rows that are neither switched nor quiescent, but "active." The states could be born, for instance, when $I_{\mathrm{dc}}$ is increased so that vortices start to enter a $Q$ region but not strongly enough to switch it. Junctions in the active rows undergo highly nonlinear oscillations, and propagating vortices are localized. These states create additional steps in the $I-V$ characteristics between two RS steps, and are detectable. Thus, they should be considered for a comprehensive treatment of row switching.

Apart from investigating the RS states, we have introduced in this article a systematic approach to the analysis of the dynamics of 2D Josephson arrays. Unlike 1D arrays, which have already led to a great amount of insight into important phenomena (such as soliton propagation and interaction in the parallel-connected arrays, ${ }^{41,50}$ or synchroniza- 


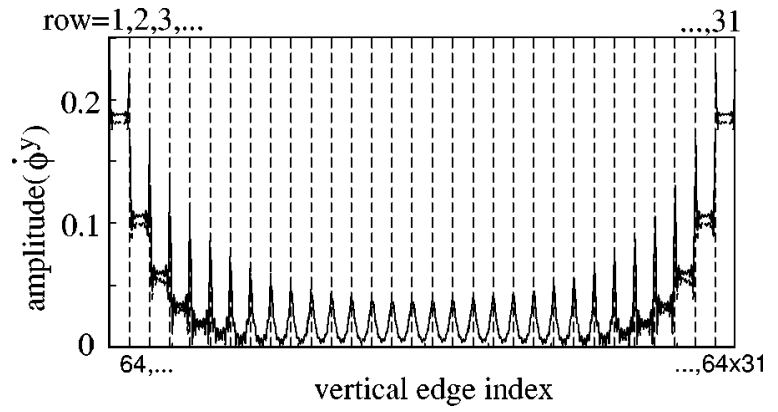

FIG. 13. Dimensionless ac voltage amplitudes $\dot{\phi}^{y}$ for pattern 1 in a large array of size $N^{x}=63$ and $N^{y}=31$. The other parameters are the ones used in pattern 1 in Fig. 9. The amplitudes decay quickly from the boundaries, and nearly vanish inside the array. The tendency was already present in Fig. 9 for the $31 \times 7$ array, but it is clearer here. Consequently, were it to be used as an oscillator, the total ac output voltage for this pattern would not scale favorably with increasing size.

tion, clustering, and magnetlike phase transitions in the series-connected arrays ${ }^{16,40}$ ), 2D arrays have been much harder to analyze. This is partly due to their network equations being more complicated, and also to their having a wider variety of solutions. As our weakly nonlinear analysis shows, the difficulty regarding the formulation is reduced by the compact mesh formalism introduced in previous numerical studies. ${ }^{27,29,4,31,32,18}$ We feel that the transparent form of the mesh equations has the potential to provide analytical information in the strongly nonlinear regime.

Of these strongly nonlinear solutions, two are of particular interest. First, coherent states, such as pattern 1 in Fig. 2, might be suitable for oscillator applications, if $f$ is kept small so that the whole array operates nearly in phase. However, for the completely row-switched state to be useful, overdamped junctions, which rotate less smoothly and, thus, produce larger ac amplitudes, should be employed. ${ }^{6}$ The extension of our analysis for this case (concerning only pattern 1) appears to be straightforward. However, we can already point out a complication due to the spatial distribution of the ac amplitudes. Recall how the $\phi^{y}$ amplitudes in pattern 1 (Fig. 9) decay from the boundaries into the interior of the array. This effect is more clearly illustrated in Fig. 13 where the ac amplitudes are computed for pattern 1 in a larger array $\left(N^{x}=63\right.$ and $\left.N^{y}=31\right)$, the other parameters being identical. The amplitudes decay quickly, and nearly vanish inside the array. Consequently, the total ac voltage does not increase significantly even when more junctions are interconnected.

Finally, flux flow ${ }^{37,32,18}$ is also a highly nonlinear but disordered regime in which localized vortices propagate "diffusively.' Theoretical studies so far have been based on phenomenological pictures of vortices and their interactions. ${ }^{3} \mathrm{~A}$ more formal treatment of these solutions and a detailed prediction of, for instance, the flux-flow resistance is strongly awaited both from the theoretical and experimental points of view.

\section{ACKNOWLEDGMENTS}

We thank Steve Strogatz for helpful guidance throughout the course of this project. Herre van der Zant and Terry
Orlando encouraged our studying the row-switching phenomenon, and taught us the necessary background on Josephson arrays. We especially thank Enrique Trías for sharing with us his unpublished experimental results and simulations, computer codes, and general knowledge of the field. We also benefited from valuable discussions on formulation and computational issues with Joel Phillips and Jacob White. Fruitful conversations with Amy Duwel and Mac Beasley are also acknowledged. Although the calculations presented here have been carried out on standard workstations, preliminary computations were performed at MIT on the Connection Machine 5, project SCOUT (ARPA Contract No. MDA972-92-J-1032). Research supported in part by NSF Grants No. DMR-9610042 (through Terry Orlando) and DMS-9500948 (through Steve Strogatz). M.B. gratefully acknowledges the financial support from the Ministerio de Educación y Cultura of Spain.

\section{APPENDIX: SMALL ASPECT-RATIO APPROXIMATION}

Following the large aspect-ratio approximation presented in Sec. III B 1, we calculate now a bulk approximation to the dc equations (29)-(32) in a $Q$ region with a small aspect ratio, i.e., when its vertical size $N^{y}$ is much larger than $N^{x}$. Far from the top and bottom boundaries, the solution is expected to be independent of $j$ (assuming there are no trapped vortices). Then, the dc equations (29),(30) simplify to

$$
\begin{gathered}
\sin \overline{\phi_{0}^{x}}(i)=0, \quad \text { thus } \overline{\phi_{0}^{x}}(i)=0, \\
\overline{\phi_{0}^{y}}(i+1)-\overline{\phi_{0}^{y}}(i)=-2 \pi f .
\end{gathered}
$$

From Eq. (32) and the boundary conditions (37) we can construct the following telescope sum which must be satisfied:

$$
\sum_{i=1}^{N^{x}+1} \sin \overline{\phi_{0}^{y}}(i)=\left(N^{x}+1\right) I_{\mathrm{dc}} .
$$

From these two equations (A2),(A3) we can then solve for the vertical phases in the bulk of the $Q$ region:

$$
\overline{\phi_{0}^{y}}(i)=2 \pi f\left(\frac{N^{x}}{2}+1-i\right)+\alpha,
$$

where

$$
\alpha=\arcsin \left\{\frac{\left(N^{x}+1\right) \sin (\pi f)}{\sin \left[\left(N^{x}+1\right) \pi f\right]} I_{\mathrm{dc}}\right\} .
$$

Compare this with the large aspect-ratio case (47) in which $\overline{\phi_{0}^{y}}=\arcsin I_{\mathrm{dc}}$ is independent of $i$ in the bulk of a $Q$ region. In contrast, in the present small aspect-ratio case, the external field $f$ is absorbed now by the vertical junctions in order to ensure the flux quantization restriction (30). Note also that consideration of the top and bottom edges is crucial to introduce matching across the switched regions or to the array boundaries, but is neglected here. Without the correction from the edges, phase relations across the $S$ rows are not 
well defined. However, the small aspect-ratio approximation is still significant because it provides a clue to an important question: what is the lower bound for a $Q$ region to remain unbroken? Thus, we use this calculation when we discuss the existence and stability of RS patterns in Sec. V. In this context, the small aspect-ratio approximation is the limiting case for which a $Q$ region is most easily broken by raising either $f$ or $I_{\mathrm{dc}}$.
*Present address: Institute for Mathematics \& its Applications (IMA), University of Minnesota, Minneapolis, MN 55455; shinya@ima.umn.edu

${ }^{1}$ H.S.J. van der Zant, L.J. Geerligs, and J.E. Mooij, Europhys. Lett. 19, 541 (1992); H.S.J. van der Zant, F.C. Fritschy, W.J. Elion, L.J. Geerligs, and J.E. Mooij, Phys. Rev. Lett. 69, 2971 (1992).

${ }^{2}$ U. Geigenmüller, C.J. Lobb, and C.B. Whan, Phys. Rev. B 47, 348 (1993).

${ }^{3}$ T.P. Orlando, J.E. Mooij, and H.S.J. van der Zant, Phys. Rev. B 43, 10218 (1991): H.S.J. van der Zant, F.C. Fritschy, T.P. Orlando, and J.E. Mooij, ibid. 47, 295 (1993).

${ }^{4}$ D. Domínguez and J.V. José, Phys. Rev. Lett. 69, 514 (1992); Phys. Rev. B 53, 11692 (1996).

${ }^{5}$ T.J. Hagenaars, P.H.E. Tiesinga, J.E. van Himbergen, and J.V. Jose, Phys. Rev. B 50, 1143 (1994).

${ }^{6}$ K. Wiesenfeld, S.P. Benz, and P.A.A. Booi, J. Appl. Phys. 76, 3835 (1994).

${ }^{7}$ A.S. Landsberg, Y. Braiman, and K. Wiesenfeld, Phys. Rev. B 52, 15458 (1995).

${ }^{8}$ G. Filatrella and K. Wiesenfeld, J. Appl. Phys. 78, 1878 (1995).

${ }^{9}$ J. Oppenländer, G. Dangelmayr, and W. Güttinger, Phys. Rev. B 54, 1213 (1996).

${ }^{10}$ I.F. Marino and T.C. Halsey, Phys. Rev. B 50, 6289 (1994); I.F. Marino, ibid. 55, 551 (1997).

${ }^{11}$ R. Bhagavatula, C. Ebner, and C. Jayaprakash, Phys. Rev. B 45, 4774 (1992); 50, 9376 (1994).

${ }^{12}$ Proceedings of the ICTP Workshop, Trieste, Aug. 7-11, 1995, edited by H.A. Cerdeira and S.R. Shenoy [Physica B 222, 4 (1996)].

${ }^{13}$ S.P. Benz and C.J. Burroughs, Supercond. Sci. Technol. 4, 561 (1991); P.A.A. Booi and S.P. Benz, Appl. Phys. Lett. 64, 2163 (1994).

${ }^{14}$ A. Petraglia, N.F. Pedersen, P.L. Christiansen, and A.V. Ustinov, Phys. Rev. B 55, 8490 (1997).

${ }^{15}$ A.E. Duwel, E. Trías, T.P. Orlando, H.S.J. van der Zant, S. Watanabe, and S.H. Strogatz, J. Appl. Phys. 79, 7864 (1996); A.E. Duwel, S. Watanabe, E. Trías, T.P. Orlando, H.S.J. van der Zant, and S.H. Strogatz, ibid. 82, 4661 (1997).

${ }^{16}$ P. Hadley, M.R. Beasley, and K. Wiesenfeld, Phys. Rev. B 38, 8712 (1988); S. Watanabe and S.H. Strogatz, Physica D 74, 197 (1994); K. Wiesenfeld and J.W. Swift, Phys. Rev. E 51, 1020 (1995); K. Wiesenfeld, P. Colet, and S.H. Strogatz, Phys. Rev. Lett. 76, 404 (1996).

${ }^{17}$ H.S.J. van der Zant, C.J. Müller, L.J. Geerligs, C.J.P.M. Harmans, and J.E. Mooij, Phys. Rev. B 38, 5154 (1988); T.S. Tighe, A.T. Johnson, and M. Tinkham, ibid. 44, 10286 (1991).

${ }^{18}$ E. Trías, M.S. thesis, MIT, 1995; E. Trías (unpublished).

${ }^{19}$ E. Trías, M. Barahona, T.P. Orlando, and H.S.J. van der Zant, IEEE Trans. Appl. Supercond. 7, 3103 (1997).

${ }^{20}$ S.G. Lachenmann, T. Doderer, D. Hoffmann, R.P. Huebener, P.A.A. Booi, and S.P. Benz, Phys. Rev. B 50, 3158 (1994); S.G.
Lachenmann, T. Doederer, and R.P. Huebener, ibid. 53, 14541 (1996).

${ }^{21}$ H.S.J. van der Zant, F.C. Fritschy, T.P. Orlando, and J.E. Mooij, Europhys. Lett. 18, 343 (1992).

${ }^{22}$ W. Yu and D. Stroud, Phys. Rev. B 46, 14005 (1992); W. Yu, K.H. Lee, and D. Stroud, ibid. 47, 5906 (1993).

${ }^{23}$ A. Brass, H.J. Jensen, and A.J. Berlinsky, Phys. Rev. B 39, 102 (1989); T. Matsuda, K. Harada, H. Kasai, O. Kamimura, and A. Tonomura, Science 271, 1393 (1996).

${ }^{24}$ R. Kleiner, F. Steinmeyer, G. Kunkel, and P. Müller, Phys. Rev. Lett. 68, 2394 (1992).

${ }^{25}$ S.G. Lachenmann, T. Doderer, R.P. Huebener, T.J. Hagenaars, J.E. van Himbergen, P.H.E. Tiesinga, and J.V. José, Phys. Rev. B 56, 5564 (1997).

${ }^{26}$ M. Barahona, E. Trías, T.P. Orlando, A.E. Duwel, H.S.J. van der Zant, S. Watanabe, and S.H. Strogatz, Phys. Rev. B 55, R11 989 (1997).

${ }^{27}$ K. Nakajima and Y. Sawada, J. Appl. Phys. 52, 5732 (1981).

${ }^{28}$ J.S. Chung, K.H. Lee, and D. Stroud, Phys. Rev. B 40, 6570 (1989).

${ }^{29}$ A. Majhofer, T. Wolf, and W. Dieterich, Phys. Rev. B 44, 9634 (1991); D. Reinel, W. Dieterich, T. Wolf, and A. Majhofer, ibid. 49, 9118 (1994).

${ }^{30}$ F. Falo, A.R. Bishop, and P.S. Lomdahl, Phys. Rev. B 41, 10983 (1990); N. Grønbech-Jensen, A.R. Bishop, F. Falo, and P.S. Lomdahl, ibid. 45, 10139 (1992).

${ }^{31}$ J.R. Phillips, H.S.J. van der Zant, J. White, and T.P. Orlando, Phys. Rev. B 47, 5219 (1993).

${ }^{32}$ J.R. Phillips, H.S.J. van der Zant, and T.P. Orlando, Phys. Rev. B 50, 9380 (1994).

${ }^{33}$ A. Petraglia, F. Filatrella, and G. Rotoli, Phys. Rev. B 53, 2732 (1996).

${ }^{34}$ B.H. Larsen and S.P. Benz, Appl. Phys. Lett. 66, 3209 (1995).

${ }^{35}$ K.K. Likharev, Dynamics of Josephson Junctions and Circuits (Gordon and Breach, New York, 1986).

${ }^{36}$ S.H. Strogatz, Nonlinear Dynamics and Chaos: with Applications to Physics, Biology, Chemistry, and Engineering (AddisonWesley, Reading, MA, 1994).

${ }^{37}$ T.P. Orlando and K.A. Delin, Foundations of Applied Superconductivity (Addison-Wesley, Reading, MA, 1991); M. Tinkham, Introduction to Superconductivity (2nd ed.) (McGraw-Hill, New York, 1996).

${ }^{38}$ The choice of the current distribution $I_{\text {ext }}$ may influence the results when inductance matrices are truncated. See Ref. 4.

${ }^{39} \mathrm{G}$. Strang, Introduction to Applied Mathematics (WellesleyCambridge Press, Wellesley, MA, 1986).

${ }^{40}$ S. Watanabe and J. Swift, J. Nonlinear Sci. 7, 503 (1997).

${ }^{41}$ S. Watanabe, H.S.J. van der Zant, S.H. Strogatz, and T.P. Orlando, Physica D 97, 429 (1996).

${ }^{42}$ The other roots are possible, but this is the root we observe.

${ }^{43}$ We note that Eq. (38) ensues from the open lateral boundary conditions. If the right and left boundaries are connected to ob- 
tain periodic boundary conditions (Refs. 22, 2, and 10) the mesh currents $\psi$ do not necessarily vanish in the $S$ rows, and qualitative changes to the results are expected. We have not studied this case.

${ }^{44} \mathrm{~S}$. Watanabe (unpublished).

${ }^{45}$ S. Teitel and C. Jayaprakash, Phys. Rev. B 27, 598 (1983).

${ }^{46}$ M. Barahona (unpublished); See, also, Ref. 47.
${ }^{47}$ M. Barahona, S.H. Strogatz, and T.P. Orlando, Phys. Rev. B 57, 1181 (1998); M. Barahona, Ph.D. thesis, MIT, 1996.

${ }^{48}$ For ladder arrays with a single column and $N^{y}$ rows, see J. Kim, W.G. Choe, S. Kim, and H.J. Lee, Phys. Rev. B 49, 459 (1994).

${ }^{49}$ J. Oppenländer (unpublished).

${ }^{50}$ N.F. Pedersen and A.V. Ustinov, Supercond. Sci. Technol. 8, 389 (1995). 